\title{
Developing Retailer Selection Factors for Collaborative Planning, Forecasting and Replenishment
}

Panaihfar, F., Heavey, C, \& Byrne, PJ. 2015. Developing Retailer Selection Factors for Collaborative Planning, Forecasting and Replenishment. INDUSTRIAL MANAGEMENT \& DATA SYSTEMS, 115, 7, pp1292-1324.

\begin{abstract}
Purpose- Selecting an appropriate partner is a vital and strategic decision-making process in any supply chain collaboration initiative. The purpose of this study is to introduce and explore the key factors considered by manufacturers in the selection of an appropriate retailer(s) for Collaboration and Collaborative Planning, Forecasting and Replenishment (CPFR) implementation and the relationships between these factors.

Design/methodology/approach - A comprehensive literature review and experts' views are applied to identify the main retailer selection and evaluation factors for CPFR implementation. A Fuzzy DEMATEL approach is then used to rank and analysis the interaction among identified factors. The findings are finally evaluated using a case study from a high-tech industry.

Findings- The most important partner selection factors comprising of five dimensions and 24 factors are introduced. Of the identified criteria, three factors: manufacturer's familiarity with the retailer, workforce skills and training and customer service orientation and capability have been identified as critical when selecting retailers for CPFR implementation. The technological capabilities dimensions are identified as the only net cause dimension which affects all other dimensions and its importance and role in simplifying and enhancing the speed and flexibility of CPFR implementation.

Practical implications- The study identifies practical retailer selection factors for CPFR implementation and the causal relationships between factors. Developed retailer selection dimensions and criteria will assist manufacturers and retailers in understanding the role these factors play in CPFR implementation. This will also assist in appropriate retailer(s) selection by manufacturers.

Originality/value - This study contributes to the literature on CPFR and tackles the important issue of selecting appropriate partners by developing retailer selection dimensions and criteria in CPFR implementation.
\end{abstract}

Keywords CPFR implementation; partnership; retailer selection factor; Fuzzy DEMATEL

Paper type Research paper

\section{Introduction}

Collaboration and Collaborative Planning, Forecasting and Replenishment (CPFR) in the context of supply chains have been well discussed in previous research (Holmström et al. 2002; Sahay 2003; Daugherty et al. 2006; VICS 2013; Byrne and Heavey 2006; Thomé and Hollmann 2014; Panahifar et al. 2014; Panahifar et al. 2015b). It is well recognised that collaboration between supply chain members can facilitate enhanced strategic and operational focus, thus allowing individual organisations to better exploit their core competencies (Daugherty et al. 2006). Sahay (2003) identified three major types of collaborative relationships: manufacturing/supplier collaboration; manufacturing/customer (i.e. retailers) collaboration and collaboration with third and fourth party logistics providers, with the manufacturer-retailer collaboration identified as the most significant. Sahay (2003, p. 77) argued that "the main focus in this collaboration is developing an understanding of demand at the point of consumption, followed by the creation of a mutually agreed replenishment plan". CPFR has also been acknowledged as one of the most important collaborative initiatives in business to business commerce potentially leading to radically reduced inventories and expenses while simultaneously improving customer service (VICS 2013). Although promising results have been presented in relation to CPFR applications, significant implementation challenges still exist, which has led to slower than expected uptake rates (Panahifar et al. 2015b). 
A review of the importance of partner selection in successful collaboration practices suggests that there is a strong correlation between the selection of the most suited partner(s) and the main barriers to a successful collaborative approach, including: lack of trust - Min et al. 2005; lack of compatibility of partners' abilities - Panahifar et al. 2014; cultural conflicts - Kelly et al. 2002. In general, improper partner selection is recognised as one the main reasons for below standard performance in trading partnerships (Ireland et al. 2002). Whilst it is recognised that selection of appropriate partners is a critical, complex and time consuming task in CPFR (Sheffi, 2002; Fu et al. 2010), it has been under represented in the academic literature to date with only a small number of papers covering the topic (Chung and Leung, 2005, Panahifar et al. 2014). This has led to a gap in the knowledge base relating to the identification and selection of appropriate implementation partners so as to maximise the likelihood of CPFR success. This study explicitly addresses this gap through retailer selection factor analysis for CPFR, including factor identification, ranking and interaction identification using a hybrid approach including expert opinion. A review of previous literature shows that while there are many partner selection factors affecting collaboration, from a practice based perspective more efforts should be made to include expert opinion in the evaluation and distillation of such factors. In this study, this process uses a detailed literature review to identify the most significant factors which positively affect a manufacturer's ability to collaborate with retailers. In order to build on this and to capture practice based omissions from the literature, expert views were then used to assess the identified factors and to alter and adapt as appropriate based on their practice expertise.

Traditionally, Multi Criteria Decision Making (MCDM) methods have been widely used in partner selection, (Huang and Keskar, 2007). In this study a hybrid version of one such technique is used to evaluate the relevant partner selection factors, the DEMATEL (Decision-making trial and evaluation laboratory) method. The DEMATEL model has been successfully applied in many fields and areas, such as outsourcing, project management and marketing including the use of Fuzzy DEMATEL in partner selection and evaluation studies (Chang et al. 2011; Liou, 2012).

The remainder of this study is organized as follows. Section 2 presents the methodology by which this study was completed, expanding on factor selection and refinement through literature review and expert opinion, followed by Fuzzy DEMATEL evaluation and concluding with a case validation. Following this, Section 3 presents results for each of the methodological steps and discusses the industrial validation of the results in the high tech industry case organisation. Sections 4 and 5, respectively discusses the results followed by concluding remarks identifying their theoretical and practical implications.

\section{Methodology}

High-tech industries such as semiconductor, computer and peripheral equipment, telecommunications, pharmaceutical and medical devices are characterized by rapid change, intense competition, and a highly uncertain environment (Huang and Lin 2006). These characteristics lead to severe difficulties in relation to demand forecasting for high-tech products even in what can be regarded as stable economies (Wu et al 2005), leading to organisational underperformance. In such instances, high levels of collaboration between supply chain partners (e.g. CPFR) have been proposed as a possible method for addressing this issue (Washida 2005; Yuan et al. 2010). In recognition of the value of CPFR in this domain, Panahifar et al (2015c) found that of the presented literature on the topic, the high tech industry was most frequently represented. Based on the relevance and importance of CPFR in this field this study focuses its analytical lens on the high tech industry and the role of retailer selection factors in CPFR implementation. 
This study focuses significantly on expert opinion and perception and the presented methodology supports this. In particular the study includes four supporting techniques: literature review, structural interviews, survey using fuzzy techniques and case study. The general methodology and fuzzy DEMATEL workflow is outlined in Figure 1. The labelling of Figure 1 represents the sections under which each component of the methodology is discussed (e.g. Section 2.1, 2.2 and 2.3). In the process of selecting and refining retailer selection factors, the study initially utilises a literature review to develop the CPFR retailer selection factor base which is then refined using expert opinion. Following the refinement of the retailer selection factors, a Fuzzy DEMATEL approach is used to identify and assess the main dimensions and criteria for CPFR retailer selection. The model and its findings were then subjected to an industrial case evaluation in a high tech organisation. The Fuzzy DEMATEL steps used in this study are extended from (Panahifar et al. 2015a) and have been derived and updated based on approaches presented by Lin and $\mathrm{Wu}$ (2008) and Chang et al (2011). The following sub sections present the main steps of the methodology, beginning with retailer selection factor identification and refinement.

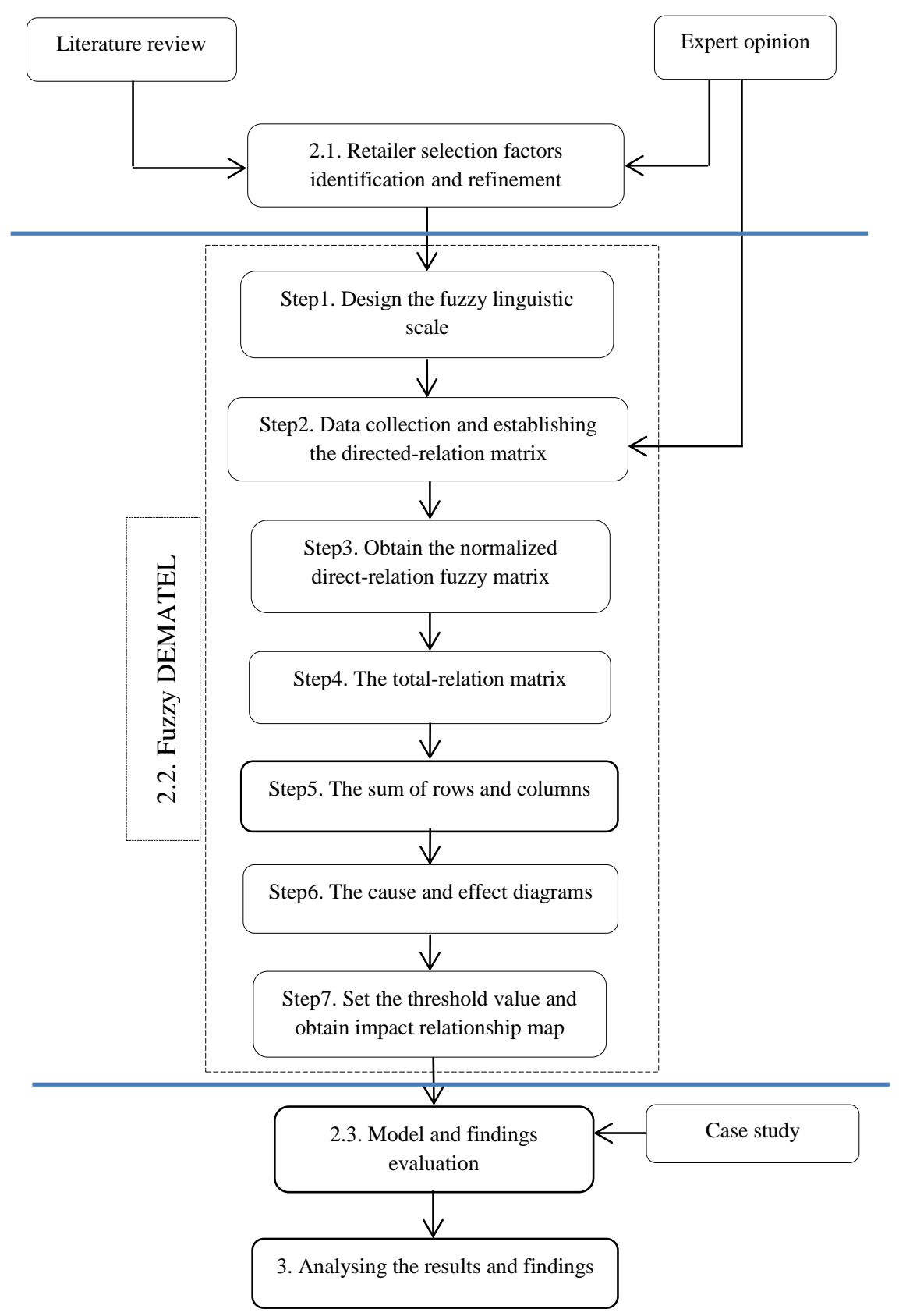


Figure 1: Research methodology

\subsection{Retailer selection factor identification and refinement}

To identify an initial list of retailer selection factors a detailed literature review was completed focusing specifically on concepts relating to CPFR and collaboration partner selection, in order to identify factors which 1) have been identified as important partner selection factors for CPFR implementation, and 2) affect a partner's ability for collaboration and information sharing with others. The key words 'CPFR', 'collaboration' and 'partner selection' in article titles were used to search the scholarly databases of ISI Web of Science ${ }^{\circledR}$, Taylor \& Francis, Google Scholar ${ }^{\mathrm{TM}}$ and Emerald from the year 1998 (when CPFR was first academically documented) up to 2014. This search resulted in the collection of 95 scholarly articles (Panahifar et al. 2015c). These 95 articles were then critically evaluated to identify relevant partner selection factors for collaboration in general and particularly with respect to CPFR implementation. This process involved two separate analytical runs for factor analysis. In the first run, based on the key criterion of partner selection, papers were identified and classified. In the second run, a more detailed analysis was conducted, which involved a comprehensively assessment for each of the selected papers for the purpose of data relevant data extraction (e.g. selection factors and dimensions).

Following the extraction of selection factors and dimensions from the literature, expert opinion was engaged to refine the list through the identification of additional factors not captured in existing literature and to eliminate or combine non relevant or similar factors. This step is critical in ensuring the input factors being presented to the Fuzzy DEMATEL model are relevant (for practice) with the removal of non-relevant factors preventing model bias. For this, a three experts were brought together to form a working group. Each of these experts were chosen due to their role as supply chain directors, their involvement in CPFR implementation projects and their relevant backgrounds in the high-tech sector. To complete this task, this expert group were specifically tasked with the following sub-steps:

- Literature review factor analysis: The purpose was to review the general objectives and findings of the literature study. In this phase, detailed definitions for each of the identified factors from the literature were presented to the experts group to ensure a definitive common understanding of the factors presented.

- Factor addition: Using the list of factors identified in the literature with accompanying definitions, the expert group was tasked with the identification and inclusion of any factors not represented on the list based on their practical experience.

- Factor elimination: The expert group were then tasked with making any necessary adjustments to the list of factors by eliminating or integrating similar dimensions and criteria. In this step they were also tasked with term refinement where necessary.

- Factor grouping: Finally the expert group was tasked with discussing factor grouping based on proposed literature dimensions and to suggest new dimensions if appropriate.

To complete these steps the nominal group technique (NGT) was used to generate a consensus position in the selection of the most important factors related to the CPFR implementation process. The NGT is a powerful learning and development tool developed by Delbecq et al (1975) to facilitate effective group decision-making. The purpose of the NGT technique is to generate information in response to an issue that can then be prioritised by a group of experts (Potter et al 2004). To facilitate this process, the NGT session was opened with a trigger question "what are the important retailer selection factors for adopting CPFR in high-tech industries'? The NGT session then followed with the 
generation of individual ideas; exchanging lists of factors; collective identification of headings for factor categorisation; and editing until group consensus was reached.

Following the consolidation of the list of factors and engagement in the NGT session, the next step involved the development of a questionnaire, including all factors selected from the previous step, for the purpose of identifying the influence one factor on the list has through a series of pairwise comparisons over the others using a five point scale from "No influence" to "Very high influence". The questionnaire was pretested by two CPFR experts. The pre-test was conducted via email and its objective was to ensure the ambiguity, clarity and appropriateness of the items used to operationalize each of the factors. Based on the feedback received from these two experts, the questionnaire was modified to enhance clarity and appropriateness of the measures purporting.

This questionnaire was then distributed to a group of experts with extensive knowledge and experience of CPFR implementation for the purpose of identifying the most significant and dominant retailer selection factors using the fuzzy DEMATEL model. Of those contacted, 12 CPFR experts agreed to partake in the study having been approached prior to completing the study to ascertain their willingness to attend due to the time consuming nature of the task. Each of these 12 experts are either industrial practitioners with an average of 16 years of experiences in international high tech industries or academic scholars with research concentrations in the supply chain management domain. Summary details on each of the experts are presented in Table 1. The pretested questionnaires were emailed to each of the expert participants and once completed, emailed back to the investigators. In order to capture more effective and significant factors from retailer selection situations, expert participants were encouraged to recall their most recent CPFR implementation with their trading partners.

Table 1. Expert Participants

\begin{tabular}{|c|c|c|c|c|}
\hline Experts & High-tech sector & $\begin{array}{l}\text { Academia/ } \\
\text { Industry }\end{array}$ & $\begin{array}{l}\text { Background/ } \\
\text { Current position }\end{array}$ & $\begin{array}{l}\text { Years of } \\
\text { experiences }\end{array}$ \\
\hline E1 & & $\bar{I}$ & $\begin{array}{l}\text { Senior Executive of Supply Chain } \\
\text { global programmes }\end{array}$ & 27 \\
\hline E2 & & $A \& I$ & $\begin{array}{l}\text { R \& D manager (New Production } \\
\text { Technologies) }\end{array}$ & 22 \\
\hline E3 & $\begin{array}{l}\text { Computer and } \\
\text { digital equipment }\end{array}$ & I & $\begin{array}{l}\text { Director of Supply Chain Business } \\
\text { Intelligence }\end{array}$ & 19 \\
\hline $\mathrm{E} 4$ & & I & Quality Manager & 10 \\
\hline E5 & & $A \& I$ & $\begin{array}{l}\text { Supply chain lecturer and } \\
\text { researcher }\end{array}$ & 10 \\
\hline E6 & & I & Sales department & 12 \\
\hline E7 & & I & Production planning and operations & 7 \\
\hline E8 & Automotive & I & Cost engineering & 10 \\
\hline E9 & & $A \& I$ & $\begin{array}{l}\text { Supply chain lecturer and } \\
\text { researcher }\end{array}$ & 11 \\
\hline E10 & Medical Devices & I & Quality Manager & 27 \\
\hline E11 & Semiconductors & I & Senior Director of Operations & 23 \\
\hline E12 & $\begin{array}{l}\text { Mobile phone and } \\
\text { communications } \\
\text { Equipment }\end{array}$ & $\mathrm{I}$ & Operations engineer & 16 \\
\hline
\end{tabular}

\subsection{Fuzzy DEMATEL}

In this study fuzzy DEMATEL (See Figure 1) has been used as an MCDM technique for the purpose of taking an input list of retailer selection factors and translating these into a framework which clearly presents the interrelationships between these factors. In this study the mathematical workings of each of the seven sub steps are outlined in this section with summarised results from each step presented in 
Section 3. The end product of the Fuzzy DEMATEL process is a visual representation that one can use to represent a structural model which shows the relationship between the causes and effects of criteria.

\section{Step 1: Design the fuzzy linguistic scale}

To deal with the ambiguities of human assessments, fuzzy linguistic scales are used which expresses different degrees of 'influence' and their corresponding positive triangular fuzzy numbers.

\section{Step 2: Data collection and establishing the directed-relation matrix}

This study surveys experts with extensive knowledge and experiences of CPFR implementation in high-tech industries using questionnaires. To measure the relationship between criteria $C=\left\{C_{i} \mid i=1\right.$, $2, \ldots, \mathrm{n}\}$, a decision group of $p$ experts was asked to make sets of pair-wise comparisons in terms of linguistic terms. This helps to transform triangular fuzzy numbers into the initial direct-relation matrix. Hence, $p$ fuzzy matrices $\tilde{Z}^{1}, \tilde{Z}^{2}, \ldots, \tilde{Z}^{p}$ each corresponding to an expert and with triangular fuzzy numbers as its elements, are obtained. In this step, the average value of initial direct-relation matrixes is obtained from the total amount of all initial direct-relation matrixes divided by 12 (the number of respondents). Fuzzy matrix $\widetilde{Z}$ is called the initial direct-relation fuzzy matrix where:

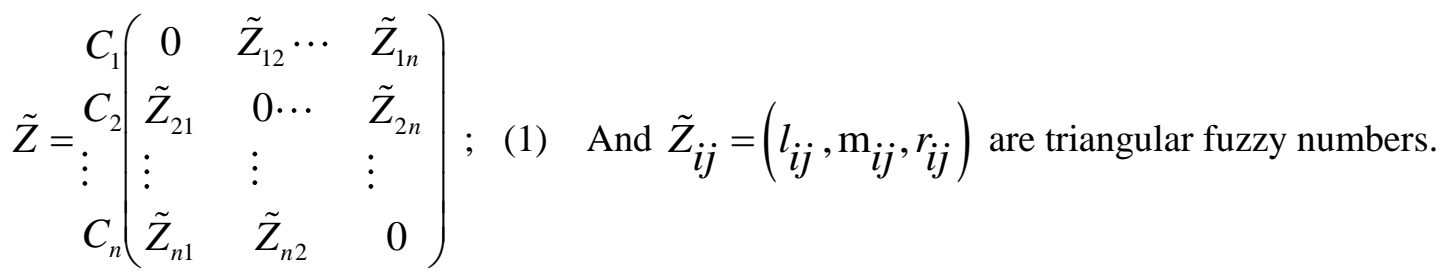

Where the following formulas are applicable to fuzzy numbers:
$k . \tilde{Z}=(k l, k m, k r)$
$\tilde{Z}_{1} \oplus \tilde{Z}_{2}=\left(l_{1}+l_{2}, m_{1}+m_{2}, r_{1}+r_{2}\right)$
$\tilde{Z}=\frac{\left(\tilde{Z}^{1}+\tilde{Z}^{2}+\ldots+\tilde{Z}^{p}\right)}{P}$

\section{Step 3: Obtain the normalized direct-relation fuzzy matrix}

On the basis of the direct-relation matrix, Equation (5), as a normalization formula is used to transform the criteria scales into comparable scales.

$$
\tilde{a}_{i j}=\sum_{j=1}^{n} \tilde{z}_{i j}=\left(\sum_{j=1}^{n} l_{i}, \sum_{j=1}^{n} m_{i}, \sum_{j=1}^{n} r_{i}\right) \text { and } \quad u=\max _{1 \leq i \leq n}\left(\sum_{i=1}^{n} r_{i j}\right)
$$

The normalized initial direction-relation fuzzy matrix is:

$$
\tilde{X}=\left(\begin{array}{lll}
\tilde{x}_{11} & \tilde{x}_{12} \cdots & \tilde{x}_{1 n} \\
\tilde{x}_{21} & \tilde{x}_{22} & \tilde{x}_{2 n} \\
\vdots & \vdots & \vdots \\
\tilde{x}_{m 1} & \tilde{x}_{m 2} \cdots & \tilde{x}_{m n}
\end{array}\right) ; \text { Where } \quad \tilde{x}_{i j}=\frac{\tilde{z}_{i j}}{u}=\left(\frac{l_{i j}}{u}, \frac{m_{i j}}{u}, \frac{r_{i j}}{u}\right)
$$




\section{Step 4: The total-relation matrix}

Once the normalized direct-relation matrix is obtained, the total-relation matrix can be acquired by using formula (8). The objective of this step is to get the overall causal relationships by computing the total-relation fuzzy matrix. To calculate the total-relation matrix $(\tilde{T})$ we assume $\lim _{k \rightarrow \infty} \tilde{x}^{k}=0$

To calculate $\tilde{x}^{k}$ we use equation 7 in order to multiple two fuzzy numbers.

$$
\tilde{N}_{1} \otimes \tilde{N}_{2} \cong\left(l_{1} \cdot l_{2}, m_{1} \cdot m_{2}, r_{1} \cdot r_{2}\right)
$$

$$
\tilde{X}_{i j}=\left(l_{i j}^{\prime}, m_{i j}^{\prime}, r_{i j}^{\prime}\right) ; X_{l}=\left(\begin{array}{lll}
0 & l_{12}^{\prime} \cdots & l_{1 n}^{\prime} \\
l_{21}^{\prime} & 0 \cdots & l_{2 n}^{\prime} \\
\vdots & \vdots & \vdots \\
l_{n 1}^{\prime} & l_{n 2}^{\prime} \cdots & 0
\end{array}\right) ; X_{m}=\left(\begin{array}{lll}
0 & m_{12}^{\prime} \cdots & m_{1 n}^{\prime} \\
m_{21}^{\prime} & 0 \cdots & m_{23}^{\prime} \\
\vdots & \vdots & \vdots \\
m_{n 1}^{\prime} & m_{n 2}^{\prime} \cdots & 0
\end{array}\right) ; X_{r}=\left(\begin{array}{lll}
0 & r_{12}^{\prime} \cdots & r_{1 n}^{\prime} \\
r_{21}^{\prime} & 0 \cdots & r_{2 n}^{\prime} \\
\vdots & \vdots & \vdots \\
r_{n 1}^{\prime} & r_{n 2}^{\prime} \cdots & 0
\end{array}\right)
$$

Then the total-relation matrix is defined by equation 8 as outlined here. The $\mathrm{I}$ is denoted as the identity matrix:

$\tilde{T}=\tilde{X}^{1}+\tilde{X}^{2}+\cdots+\tilde{X}^{k}=\tilde{X}(I-\tilde{X})^{-1}$

$\tilde{T}=\lim _{k \rightarrow \infty}\left(\tilde{X}+\tilde{X}^{2}+\cdots+\tilde{X}^{k}\right)$ Then: $\tilde{t}_{i j}=\left(l_{i j}^{\prime \prime}, m_{i j}^{\prime \prime}, r_{i j}^{\prime \prime}\right)$ and $\tilde{T}=\left(\begin{array}{lll}\tilde{t}_{11} & \tilde{t}_{12} \cdots & \tilde{t}_{1 n} \\ \tilde{t}_{21} & \tilde{t}_{22} \cdots & \tilde{t}_{2 n} \\ \vdots & \vdots & \vdots \\ \tilde{t}_{31} & \tilde{t}_{n 2} \cdots & \tilde{t}_{n n}\end{array}\right)$

According to the assumption in step 4 we have: $\lim _{k \rightarrow \infty} \tilde{X}^{k}=[0]_{n \times n}$

$$
\text { And }\left[l_{i j}^{\prime \prime}\right]=X_{l} \times\left(I-X_{l}\right) \text {; (9) }\left[m_{i j}^{\prime \prime}\right]=X_{m} \times\left(I-X_{m}\right)^{-1} ; \quad \text { (10) }\left[r_{i j}^{\prime \prime}\right]=X_{r} \times\left(I-X_{r}\right)^{-1}
$$

\section{Step 5: Obtain the sum of rows and columns}

We now calculate the relevant amounts of $\tilde{D}_{i}+\tilde{R}_{i}$ and $\tilde{D}_{i}-\tilde{R}_{i}$ whereas $\tilde{D}_{i}$ is the row sum and $\tilde{R}_{i}$ is the sum of columns in $(\tilde{T}) . \quad \tilde{T}=\left[\tilde{t}_{i j}\right], i, j \in\{1,2, \cdots, n\} ; \tilde{D}_{i}=\left(\tilde{d}_{i}\right)_{n \times 1}=\left[\sum_{j=1}^{n} \tilde{t}_{i j}\right]_{n \times 1}$ and $\tilde{R}_{i}=\left(\tilde{\mathrm{r}}_{j}\right)_{1 \times n}=\left[\sum_{i=1}^{n} \tilde{t}_{i j}\right]_{1 \times n}$

The defuzzification procedure considers the spread, height and shape of a triangular fuzzy number as important characteristics of the fuzzy number (Chang et al. 2011). The objective of defuzzification is to convert the output fuzzy variable to a crisp value. In other words, it is used to determine an exact value as the representative of the fuzzy number. To defuzzify the fuzzy weights in order to compare the alternatives in a non-fuzzy ranking method, the center of area (COA) is applied in this study using the following equation: 
$B N P=l+\frac{(r-l)+(m-l)}{3}$

The values of $\left(\tilde{\mathrm{D}}_{i}+\tilde{R}_{i}\right)^{\text {def }}$ and $\left(\tilde{\mathrm{D}}_{i}-\tilde{R}_{i}\right)^{\text {def }}$ are calculated using Equation 13.

\section{Step 6: The cause and effect diagram}

The Fuzzy DEMATEL method converts the relationship between cause and effect factors into an intelligible structural model of the system. This structural mode can propose the most important criteria which affects other criteria. In this step, a causal and effect graph is completed by mapping the dataset of (D+R, D-R). The importance of each criteria 'Prominence' is obtained by adding $D$ to $R$ which forms the horizontal axis. Similarly, the vertical axis (D-R), 'Relation' is obtained by subtracting $\mathrm{R}$ from $\mathrm{D}$, which if found positive, the group criteria is denoted a cause group, or, if the (D-R) is negative, the criteria is denoted an effect group.

\section{Step 7: Set the threshold value and obtain impact relationship map}

Setting a threshold value, $n$, to filter the obvious effects denoted by the factors is necessary in order to better explain the interrelationship among the factors and to help visualize the complex relationships between the criteria. To reduce the complexity of the impact relations map (IRM), a threshold value for the influence level will be set. Only the elements, whose influence level is higher than the threshold value, can be chosen and converted into the IRM (Liou and Chuang, 2010).

\subsection{Model and findings evaluation}

The Fuzzy DEMATEL model presents a framework for understanding the relationships between the identified retailer selection factors. This step in the process was developed to evaluate the model. To complete the evaluation exercise the results were assessed using a case organisation with the evaluation process following five sub steps:

- Case company selection;

- Evaluation of current partner selection method for collaboration and information sharing;

- Presentation of the main dimensions and factors identified;

- Discussion on the practical and managerial implications of the model and

- Discussion on perceived completeness of partner selection model.

The organisation chosen to evaluate the research findings is operating in the Medical Device industry and the group presented for validation included four divisional managers and one collaboration expert from the following departments: supply chain management; production management; quality management and process engineering. The meeting with the case organisation lasted $120 \mathrm{~min}$ and focussed on understanding and capturing partner selection factors for the purpose of collaboration and information sharing, the results of which are presented in Section 3.

\section{Results}

\subsection{Retailer selection factor identification and refinement}

Using the methodology described in Section 2.1, which included a comprehensive literature review and expert group input, 24 retailer selection factors were identified for CPFR partnering and their subsequent categorisation into 5 distinct dimensions (Table 2). In order to create the initial set of 
factors for expert evaluation, the 95 scholarly articles identified were evaluated and categorized based on their relevance in terms of CPFR partner selection or partner selection more generally. Of the papers analysed, only five factors $\left(\mathrm{C}_{2}-\right.$ high internal alignment; $\mathrm{C}_{5}$ - workforce skills and training; $\mathrm{C}_{17}$ - retailers' initiative to build trust; $\mathrm{C}_{22}$ - information security system and $\mathrm{C}_{23}$ - strong IT infrastructure) were found to have been previously identified and studied in the CPFR partnering domain (Europe ERC 2001; Lin et al. 2004; Chung and Leung 2005; Lin and Chen 2008; Fu et al. 2010). The additional 20 factors were found to have a relevance based on partner selection in collaboration and information sharing initiatives but were not directly attributable to CPFR studies. In total the literature review identified 25 factors for presentation to the expert group.

Upon completion of the literature based factor identification a compilation of relevant definitions for each of the factors was developed. These definitions, including relevant reference sources were developed to ensure a common understanding was being presented and used by each member of the expert group (Experts E1, E2 and E11 in Table 1). Factor refinement was conducted using the NGT session. Using this approach the expert group were first asked to review the 25 presented factors and based on their significant experience to identify and put forward any factors which they believed should be added. Based on this procedure the expert group agreed upon the addition of three additional factors $\left(\mathrm{C}_{14}\right.$ - footfall, $\mathrm{C}_{20}$ - inventory management and $\mathrm{C}_{24}$ - technology compatibility), bringing the list to 28 . Following factor addition the expert group were then tasked with factor elimination due to a perceived lack of relevance or factor consolidation in terms of perceived factor overlap. Based on this analysis the expert group proposed adaptations to the list leading to a final factor list of 24. The four factors which were removed or integrated with other factors in this step included retailers' on-shelf availability, ability to raise additional funding, relationship quality and company experiences.

To better categorize the final 24 factors a number of consolidating dimensions have been developed. In total, five dimensions have been derived, three of which have been previously used in the distributor selection domain: organisational and financial strengths (Cavusgil et al. 1995); marketing abilities and logistics capabilities (Lin and Chen 2008). Based on discussions with the expert group two further categorisation dimensions were added: relationship strength and technological capabilities. Finally, the 24 criteria were grouped into the five major dimensions as illustrated in Table 2.

Table 2: Retailer selection dimensions $\left(\mathrm{D}_{\mathrm{i}}\right)$ and related criteria $\left(\mathrm{C}_{\mathrm{j}}\right)$.

\begin{tabular}{ll}
\hline Organisational and Financial strengths & $\mathbf{D}_{\mathbf{1}}$ \\
\hline Management abilities and skills & $\mathrm{C}_{1}$ \\
High internal alignment & $\mathrm{C}_{2}$ \\
Flexible organisation & $\mathrm{C}_{3}$ \\
Organisational size & $\mathrm{C}_{4}$ \\
Workforce skills and training & $\mathrm{C}_{5}$ \\
Financial strength & $\mathrm{C}_{6}$ \\
Physical facilities & $\mathrm{C}_{7}$ \\
Reputation & $\mathrm{C}_{8}$ \\
& \\
\hline Marketing abilities & $\mathrm{D}_{\mathbf{2}}$ \\
\hline Sales strength & $\mathrm{C}_{9}$ \\
Market coverage & $\mathrm{C}_{10}$ \\
Familiarity with the product(s) & $\mathrm{C}_{11}$ \\
Customer service orientation and capability & $\mathrm{C}_{12}$ \\
Product compatibility & $\mathrm{C}_{13}$ \\
Footfall & $\mathrm{C}_{14}$ \\
\hline
\end{tabular}

\begin{tabular}{ll}
\hline Relationship strength & $\mathbf{D}_{\mathbf{3}}$ \\
\hline Retailers' commitment to agreed order & $\mathrm{C}_{15}$ \\
Willingness to share information & $\mathrm{C}_{16}$ \\
Retailers' initiative to build trust & $\mathrm{C}_{17}$ \\
Enthusiasm to collaboration & $\mathrm{C}_{18}$ \\
Manufacturer's familiarity with the retailer & $\mathrm{C}_{19}$ \\
\hline Logistics capabilities & $\mathbf{D}_{\mathbf{4}}$ \\
\hline Inventory management & $\mathrm{C}_{20}$ \\
On-time deliveries & $\mathrm{C}_{21}$ \\
& \\
\hline Technological capabilities & $\mathbf{D}_{\mathbf{5}}$ \\
\hline Information security system & $\mathrm{C}_{22}$ \\
Strong IT infrastructure & $\mathrm{C}_{23}$ \\
Technology compatibility & $\mathrm{C}_{24}$ \\
& \\
\hline
\end{tabular}




\subsection{Fuzzy DEMATEL}

Steps 1 and 2: Design the fuzzy linguistic scale and Data collection and establishing the directedrelation matrix

The first two steps in the fuzzy DEMATEL process (see Figure 1) involve the setting of the linguistic scale for data collection and the collection of this data in the form of a relational matrix. A five point fuzzy linguistic scale was used in this study as is presented in Table 3. Each of the twelve experts introduced in Table 1 were then presented with this linguistic scale and a blank direct-relation matrix pre populated with the 24 factors presented in Table 2. Based on this information each of the twelve experts were asked to fill in the matrix based on their own perception of the interdependences (i.e. influence) between the factors with respect to CPFR implementation projects. A sample of one completed matrix is presented in Appendix A1. Each completed direct-relation matrix was then translated into a Fuzzy matrix $Z$ using Equation 4. A sample of one completed Fuzzy matrix is presented in Appendix A2.

Table 3. The correspondence of linguistic scale and linguistic values

\begin{tabular}{lll}
\hline Linguistic scale & Linguistic values & Influence score \\
\hline No influence (No) & $(0,0,0.25)$ & 0 \\
Very low influence (VL) & $(0,0.25,0.50)$ & 1 \\
Low influence (L) & $(0.25,0.50,0.75)$ & 2 \\
High influence (H) & $(0.50,0.75,1.00)$ & 3 \\
Very high influence (VH) & $(0.75,1.00,1.00)$ & 4 \\
\hline
\end{tabular}

\section{Step 3 and 4: Obtain the normalized direct-relation fuzzy matrix and the total-relation matrix}

The next two steps are functional in nature and are part of the fuzzy DEMATEL translation process. In step 3, the normalized direct-relation matrix (Appendix A3) is developed using Equation 6 and the initial direct-relation matrix (Appendix A1). To produce the total-relation matrix, three matrices are constructed which are labelled $X_{l}, X_{m}$ and $X_{r}$. The values of $X_{l}, X_{m}$ and $X_{r}$ (Appendix A4) are calculated using the normalized initial direct-relation matrix. The values of $\left[l_{i j}^{\prime \prime}\right],\left[m_{i j}^{\prime \prime}\right]$ and $\left[r_{i j}^{\prime \prime}\right]$ will be then calculated with the help of Equations 9-11, respectively (See Appendix A5). The total-relation matrix is presented in Appendix A6.

\section{Step 5: The sum of rows and columns}

The problem under consideration in this research is to determine the level of interdependences of factors and determine their significance and their relative weight in the retailer selection for CPFR implementation. To do so, the Rank of Significance (ROS) and Rank of Impact (ROI) are calculated for each factor using $\tilde{R}_{i}, \tilde{D}_{i}, \tilde{\mathrm{D}}_{i}+\tilde{R}_{i}$ and $\tilde{\mathrm{D}}_{i}-\tilde{R}_{i}$ (Table 4) to visually simply show the position of each factor in the structural model. To complete the criteria analysis in Table 4 , all calculated $\tilde{\mathrm{D}}_{i}+\tilde{R}_{i}$ and $\tilde{\mathrm{D}}_{i}-\tilde{R}_{i}$ are defuzified using a COA (center of area) defuzification method (Equation 13). The resultant values of $\left(\tilde{\mathrm{D}}_{i}+\tilde{R}_{i}\right)^{\text {def }}$ then shows the importance of each factor and $\left(\tilde{\mathrm{D}}_{i}-\tilde{R}_{i}\right)^{\text {def }}$ assigns each factor into cause and effect groups. Table 4 also presents each of the 5 dimensions significance and impact, which is representative of the collective criteria in the grouping. 
Table 4: Rank of Significance and Impact

\begin{tabular}{|c|c|c|c|c|c|c|c|c|c|}
\hline $\begin{array}{l}\text { Dimen } \\
\text {-sion }\end{array}$ & Criteria & $\left(\tilde{\mathrm{D}}_{i}+\tilde{R}_{i}\right)^{d e f}$ & $\left(\tilde{\mathrm{D}}_{i}-\tilde{R}_{i}\right) d e f$ & $\begin{array}{c}\text { Rank of } \\
\text { Significance }\end{array}$ & $\begin{array}{l}\text { Rank of } \\
\text { Impact }\end{array}$ & $\begin{array}{l}\text { Cause/ } \\
\text { Effect }\end{array}$ & $\begin{array}{l}\text { High } \\
\text { Critical }\end{array}$ & DS & DI \\
\hline \multirow[t]{8}{*}{ D1 } & C1 & 6.067 & 0.253 & 2 & 4 & Cause & $\sqrt{ }$ & 41.536 & -0.207 \\
\hline & $\mathrm{C} 2$ & 5.290 & -0.221 & 11 & 22 & Effect & & & \\
\hline & $\mathrm{C3}$ & 5.412 & 0.017 & 9 & 9 & Cause & & & \\
\hline & $\mathrm{C} 4$ & 4.307 & -0.070 & 23 & 13 & Effect & & & \\
\hline & C5 & 5.724 & 0.311 & 3 & 2 & Cause & $\sqrt{ }$ & & \\
\hline & C6 & 5.257 & -0.103 & 13 & 18 & Effect & & & \\
\hline & C7 & 3.793 & -0.388 & 24 & 24 & Effect & & & \\
\hline & C8 & 5.686 & -0.006 & 5 & 12 & Effect & & & \\
\hline \multirow[t]{6}{*}{ D2 } & C9 & 5.720 & -0.091 & 4 & 15 & Effect & & 32.096 & -0.019 \\
\hline & $\mathrm{C} 10$ & 5.507 & -0.094 & 6 & 16 & Effect & & & \\
\hline & C11 & 5.175 & -0.146 & 15 & 19 & Effect & & & \\
\hline & $\mathrm{C} 12$ & 6.130 & 0.242 & 1 & 5 & Cause & $\sqrt{ }$ & & \\
\hline & C13 & 4.814 & 0.171 & 20 & 6 & Cause & & & \\
\hline & $\mathrm{C} 14$ & 4.750 & -0.101 & 21 & 17 & Effect & & & \\
\hline \multirow[t]{5}{*}{ D3 } & C15 & 5.251 & -0.250 & 14 & 23 & Effect & & 26.41 & -0.044 \\
\hline & C16 & 5.500 & 0.087 & 7 & 8 & Cause & & & \\
\hline & C17 & 5.274 & -0.151 & 12 & 20 & Effect & & & \\
\hline & C18 & 5.327 & -0.072 & 10 & 14 & Effect & & & \\
\hline & C19 & 5.058 & 0.342 & 17 & 1 & Cause & & & \\
\hline \multirow[t]{2}{*}{ D4 } & $\mathrm{C20}$ & 5.124 & -0.183 & 16 & 21 & Effect & & 10.57 & -0.054 \\
\hline & C21 & 5.446 & 0.129 & 8 & 7 & Cause & & & \\
\hline \multirow[t]{3}{*}{ D5 } & $\mathrm{C22}$ & 4.974 & 0.292 & 18 & 3 & Cause & & 14.44 & 0.322 \\
\hline & $\mathrm{C} 23$ & 4.925 & 0.017 & 19 & 9 & Cause & & & \\
\hline & $\mathrm{C} 24$ & 4.541 & 0.013 & 22 & 11 & Cause & & & \\
\hline
\end{tabular}

Note: DS=Dimension Significance; DI=Dimension Impact

In Table 4, the values of $\left(\tilde{\mathrm{D}}_{i}+\tilde{R}_{i}\right)^{\text {def }}$ show how significant or important each criterion is and the values of $\left(\tilde{\mathrm{D}}_{i}-\tilde{R}_{i}\right)^{\text {def }}$ divide the criteria into cause and effect groups. If the value of $\left(\tilde{\mathrm{D}}_{i}-\tilde{R}_{i}\right)^{\text {def }}$ is positive, the criteria belongs to the cause group and if its value is negative, the criteria is a member of the effect group. The ROS is determined by using the values of $\left(\tilde{\mathrm{D}}_{i}+\tilde{R}_{i}\right)^{d e f}$. From a perusal of these results it can be seen that $\mathrm{C}_{12}$ (customer service orientation and capability) is ranked as having most significant with a value of 6.130 , followed in second by $\mathrm{C}_{1}$ (management abilities and skills) with a 
value of 6.067 , and in third by $\mathrm{C}_{5}$ (workforce skills and training) with a value of 5.724. In contrast to the significance for each factor, $\mathrm{C}_{7}$ (physical facilities) and $\mathrm{C}_{15}$ (retailers' commitment to agreed order) are net receivers based on the $\left(\tilde{\mathrm{D}}_{i}-\tilde{R}_{i}\right)$ def values and the ROI and will be affected by the other factors. As $\mathrm{C}_{1}$ (management abilities and skills) has the second highest ROS, and is classified in the cause group with a relatively large value of 0.253 (ROI rank $-4^{\text {th }}$ ), it is a critical factor for the objective of retailer selection. This indicates that for the purpose of supporting collaborative planning initiatives, such as CPFR, management abilities and skills of the retailers have a strong influence on the firm and the whole chain.

The results also show that $\mathrm{C}_{19}$ (manufacturer's familiarity with the retailer) and $\mathrm{C}_{5}$ (workforce skills and training), which are ranked first and second on the ROI with values of 0.342 and 0.311 respectively, have a more influential effect on the other factors and will thus have significant potential to generate competitive advantage. In addition, $\mathrm{C}_{5}$ (workforce skills and training) has also been ranked as the third greatest significant factor with the value of 5.724. This factor is thus introduced as a critical factor in the retailer selection process because it differentiates a firm from its competitors. Based on this analysis in addition to $\mathrm{C}_{5}, \mathrm{C}_{1}$ (management abilities and skills) and $\mathrm{C}_{12}$ (Customer service orientation and capability) have been defined as highly critical factors.

From a dimension perspective it can be seen from Table 4 that organisational and financial strengths $\left(\mathrm{D}_{1}\right)$ is the most significant dimension with the largest $\left(\tilde{\mathrm{D}}_{i}+\tilde{R}_{i}\right)^{\text {def }}$ value of 41.536 whereas logistics capabilities $\left(\mathrm{D}_{4}\right)$ is the least significant dimension with the smallest value of 10.57. To further investigate the cause-effect relationship of dimensions, technological capabilities $\left(\mathrm{D}_{5}\right)$ is the only net cause based on a positive $\left(\tilde{\mathrm{D}}_{i}-\tilde{R}_{i}\right)^{\text {def }}$ value of 0.322 .

\section{Step 6: The cause and effect diagram}

Using the information from Table 4, the cause and effect diagram for the criteria and dimensions are presented in Figure 2 and Figure 3 respectively. The factors in Table 4 are mapped according to of $\left(\tilde{\mathrm{D}}_{i}+\tilde{R}_{i}\right)^{\text {def }}$ on the x-axis and $\left(\tilde{\mathrm{D}}_{i}-\tilde{R}_{i}\right)^{\text {def }}$ on the y-axis. The cause group implies the meaning of an influencing criterion, with the influence used to estimate the weights of the criteria. Factors from the cause group have a higher priority and higher intensity in relation to the effect criteria. Effect criteria receive more influence and therefore have lower priority (Tseng and Lin 2009). From a change perspective the cause criteria are more readily adapted as direct input change will lead to direct output change. The effect criteria are more difficult to influence directly and identified change activities may not result in predicted better performance. This is due to the natural influence of the cause criteria on the effect criteria. Effect criteria are by their nature directly influenced by the many cause criteria and thus less predictable in change efforts. Thus change efforts from a practice perspective are best spent on cause criteria, which if carried out correctly should also positively impact upon effect criteria. In the criteria causal diagram, the retailer selection evaluation factors are visually divided into the cause group which includes $\mathrm{C}_{1}$ (management abilities and skills), $\mathrm{C}_{3}$ (flexible organisation), $\mathrm{C}_{5}$ (workforce skills and training), $\mathrm{C}_{12}$ (customer service orientation and capability), $\mathrm{C}_{13}$ (product compatibility), $\mathrm{C}_{16}$ (willingness to share information), $\mathrm{C}_{19}$ (manufacturer's familiarity with the retailer), $\mathrm{C}_{21}$ (on-time deliveries), $\mathrm{C}_{22}$ (information security system), $\mathrm{C}_{23}$ (strong IT infrastructure) and $\mathrm{C}_{24}$ (technology compatibility). The effect group is composed of $\mathrm{C}_{2}$ (high internal alignment), $\mathrm{C}_{4}$ (organisational size), $\mathrm{C}_{6}$ (financial strength), $\mathrm{C}_{7}$ (physical facilities), $\mathrm{C}_{8}$ (reputation), $\mathrm{C}_{9}$ (sales strength), $\mathrm{C}_{10}$ (market coverage), $\mathrm{C}_{11}$ (familiarity with the product), $\mathrm{C}_{14}$ (footfall), $\mathrm{C}_{15}$ (retailers commitment to agreed 
order), $\mathrm{C}_{17}$ (retailers' initiative to build trust), $\mathrm{C}_{18}$ (enthusiasm to collaboration) and $\mathrm{C}_{20}$ (inventory management). Generally, the factors in the effect group tend to be easily impacted by others, which makes effect factors unsuitable to be a critical success factor in a partner selection process. The findings of Figure 3 are more pronounced with only the $\mathrm{D}_{5}$ (Technological Capabilities) being classified as a cause dimension.

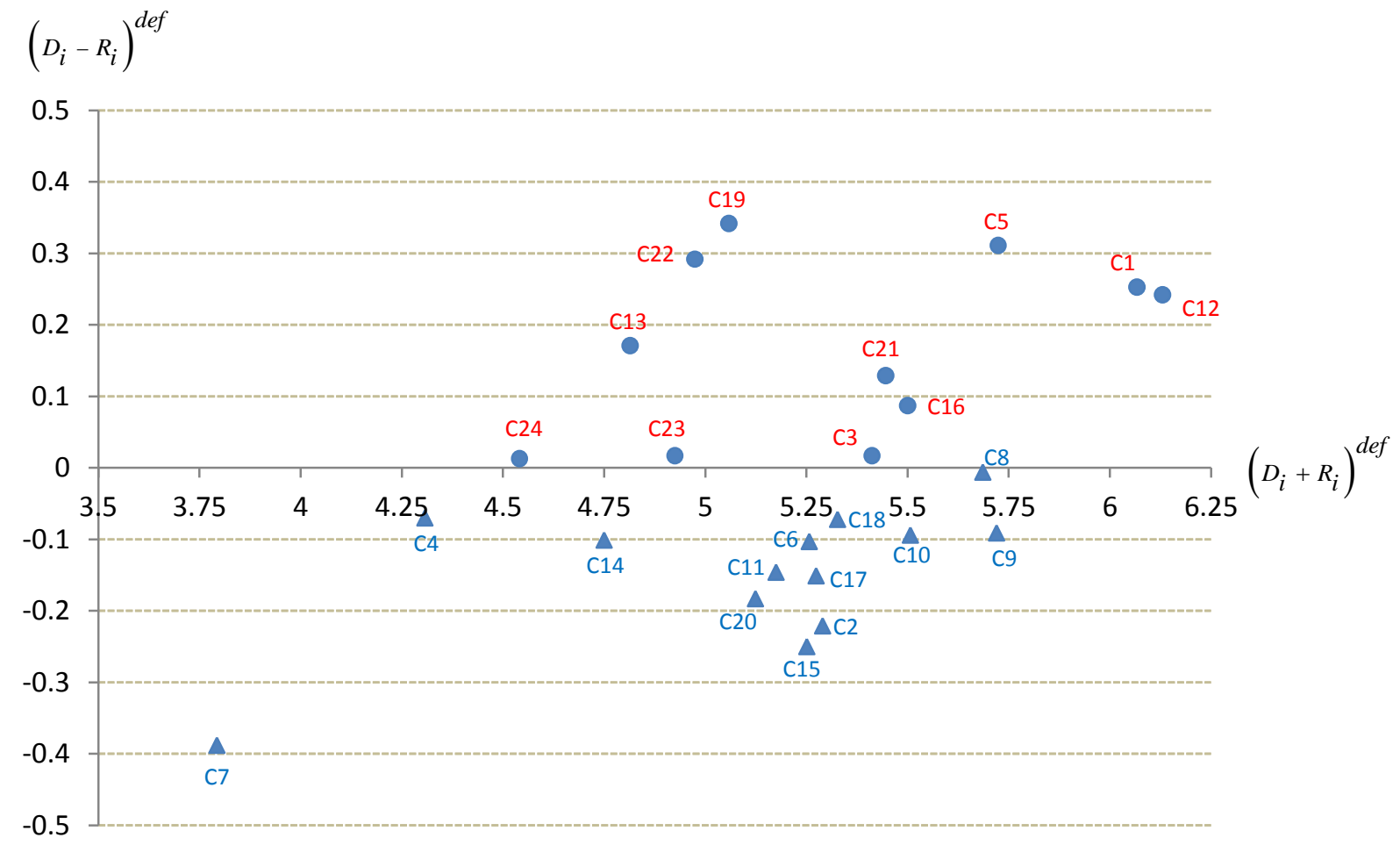

Figure 2: The cause and effect diagram of criteria (circle denoting cause and triangular denoting effect)

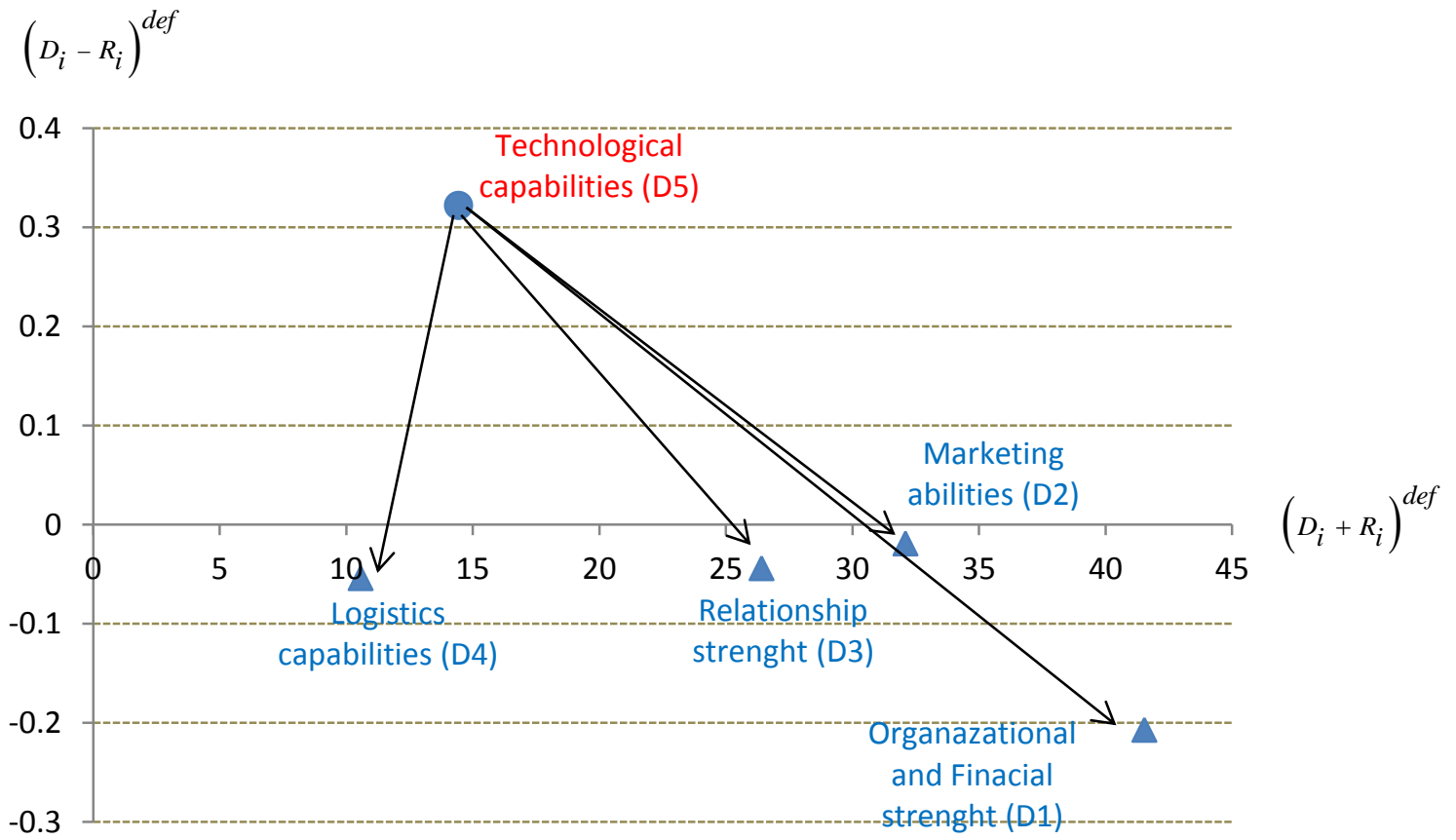

Figure 3: The cause and effect diagram of dimensions (circle denoting cause and triangular denoting effect) 


\section{Step 7: Set the threshold value and obtain the impact relation map}

The Impact Relationship Map (IRM), presented in Figure 4 was obtained, by setting a threshold value equating to the average of the positive ROI values $\left(\tilde{\mathrm{D}}_{i}-\tilde{R}_{i}\right)$ def calculated as $(\mathrm{p}=0.146)$ and selecting all factors that have an ROI in excess of this. The results show that six retailer selection factors from the cause group have a value greater than this threshold value. These elements are $C_{19}>C_{5}>C_{22}>C_{1}>$ $\mathrm{C}_{12}>\mathrm{C}_{13}$. The arrowed line linking two retailer selection factors represents their interrelationship, and the width of the line represents the intensity of the interrelationship. The thicker the line, the higher the interrelationship intensity is. As an example, factor $C_{19}$ has a high intensity with $C_{1}, C_{5}, C_{12}, C_{13}$ and $\mathrm{C}_{22}$ meaning that $\mathrm{C}_{19}$ affects all these factors intensively. The IRM helps the reader to better understand the inner dependences within a set of cause factors.

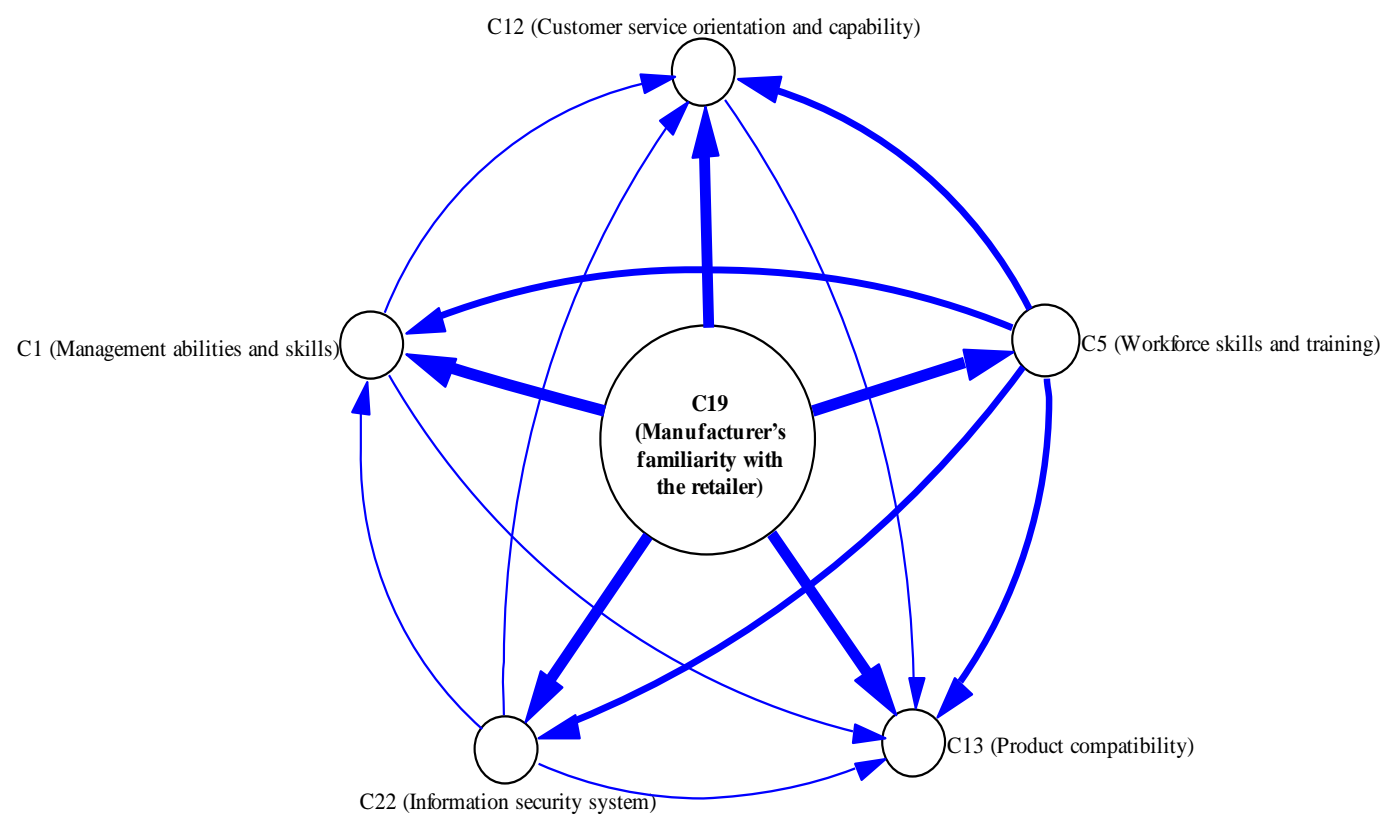

Figure 4: The impact relationship map (IRM)

\subsection{Model and findings evaluation}

In order to evaluate the veracity of the research findings the results were discussed with a Medical Device manufacturing company in Ireland, with high levels of recent collaborative activity. Results evaluation is based on presentation of the findings and acceptance (or not) of the results by the case organisation. The case organisation employs between 250 and 300 employees, and leads the distribution of nine million products per annum, seven million which they manufacture themselves and two million where manufacturing is outsourced to a number of other medical device manufacturers. The case organisation distributes these products through 10 large customers (distributers and retailers). The company have had positive collaboration experiences in recent times and have been actively continuing to grow their expertise in this domain in recent times.

The evaluation exercise was carried out onsite in the Medical Device Manufacturer and involved a collective session with the five organisational experts presented in Section 2. Prior to presentation of 
the results the current methods of partner selection for collaboration and information sharing were analysed. The case organisation currently applies a number of alternative collaborative approaches (i.e. Vender Manager Inventory and Joint Forecasting and Replenishment) and practices high levels of information sharing with its retailers and suppliers. Following this prelude, the results and findings from the study were presented, which included the presentation of the original 24 factors and their dimensional classifications followed by the results of the DEMATEL study in terms of the ROS and ROI and associated cause and effect diagrams and the impact relationship map. Upon presentation of each of these components the group were in agreement that the results were significant and comprehensive and offered significant value for manufacturers' for the effective selection of retailers for CPFR Implementation. One item of note that was brought up during the validation process was the concept of "innovative capabilities". It was suggested by the case organisation that the innovative capabilities of a retailer plays a key role in the intensity of supply chain collaboration and information sharing. This they felt was an item that could have been included in the study.

To gain insights into the practical and managerial implications of the model, the group were asked in the context of the presentation to discuss; 1) current factors used for retailer selection and evaluation for the purpose of collaboration/information sharing in the case organisation, 2) problems faced in using existing methods and techniques, and 3) whether the proposed model would enhance current practice. In general, the expert group validated the findings as an appropriate and most importantly as a comprehensive representation of standard industry practice, with all five in agreement that the proposed model is a pragmatic solution for identifying and exploring the sophisticated interactions between retailer selection factors in collaboration and information sharing process.

Analysis of the case study indicates that the model of retailer selection for CPFR implementation is in line with the practicalities of work that is conducted in industry in selecting and forming collaborative workers. The validation process established that the model's functionality would address the original business problem with retailer selection for CPFR implementation and information sharing as a general goal. The model validation group suggested that using this proposed model, companies will be better able to select and systematically evaluate their trading partners for CPFR implementation and hence prioritise manufacturing management attention to the most significant and effective factors for retailer selection. Their feedback suggested that although there are several improvements required for supporting partner selection for CPFR implementation in industry, this model significantly advances current methodologies and represents a significant proportion of the attributes involved in retailer selection and evaluation. It was also implied that a benefit of using the proposed model as a decision support tool could be in identifying appropriate retailers for collaboration/information sharing.

\section{Discussion}

Although there is an abundance of literature in the domains of collaboration and information sharing, there is a paucity of research focusing on the area of retailer selection in the context of a collaborative scheme such as CPFR. Fundamentally, strong collaborative performance is driven by strong individual entities working from a strong common platform. Incorrect partner selection can have long lasting detrimental effects. This study seeks to address a number of these issues by developing a model for the purpose of retailer selection in the CPFR implementation domain. As an indication to the need for such a study, of the papers analysed only five factors $\left(C_{2}, C_{5}, C_{17}, C_{22}\right.$ and $\left.C_{23}\right)$ were found to have been previously identified and studied in the CPFR partnering domain (Europe ERC 
2001; Lin et al. 2004; Chung and Leung 2005; Lin and Chen 2008; Fu et al. 2010. The remaining 19 factors were extracted from other related paper sources and expert input.

The model presented goes beyond a simple description of the factors and through the execution of a Fuzzy DEMATEL study collectively brings a comprehensive literature study and expert analysis to represent factor interdependencies in terms of the Rank of Impact (ROI) and Rank of Significance (ROS), both quantitatively and visually for the 24 factors identified. From comparison of the ROI and ROS, three factors including manufacturer's familiarity with the retailer $\left(\mathrm{C}_{19}\right)$, workforce skills and training $\left(\mathrm{C}_{5}\right)$ and customer service orientation and capability $\left(\mathrm{C}_{12}\right)$ have been identified as critical when selecting retailers.

The results indicate that a manufacturer's familiarity with the retailer $\left(\mathrm{C}_{19}\right)$ is the most important factor when attempting to select a retailer for a CPFR implementation project. Based on these findings, a manufacturer's familiarity with the retailer will influence other criteria significantly more than it will be influenced by others. In line with the findings of Lin and Chen (2008) and Europe ERC (2001), this suggests that CPFR should not be seen as an approach to create a good relationship with new players, but rather, it can help to enhance a good relationship with an existing trading partner. Following $\mathrm{C}_{19}$, workforce skills and training $\left(\mathrm{C}_{5}\right)$ has been identified as the next most critical criteria, based on ROI, in partner selection for CPFR implementation which is echoed in Chung and Leung (2005).

In terms of ROS, the results show that a retailer's customer service orientation and capability $\left(\mathrm{C}_{12}\right)$ is the most important factor for CPFR implementation and its improvement can lead to the amelioration of the whole system. Customer service capabilities like 'on-shelf availability' generate a better service to end users and require high level of manufacturer and retailer cooperation to succeed. This can enhance information visibility across the supply chain. As retailers are typically the closest entity to end consumers and play a significant role in estimating and forecasting real needs and demands, this can lead to significantly improved demand forecast accuracy in the entire supply chain.

Additionally, the results of this study indicate that all the three factors from the technological capabilities dimension $\left(\mathrm{D}_{1}\right)$ including $\mathrm{C}_{22}$ (information security system), $\mathrm{C}_{23}$ (strong IT infrastructure) and $\mathrm{C}_{24}$ (technology compatibility) are identified as cause factors. Technology compatibility increases organisational readiness to adopt information sharing corporations with partners. Lack of technology compatibility by a trading partner is also known as an effective barrier in information sharing practise (Viswanathan et al. 2007). Technological capabilities dimension is identified as the only net cause dimension which affects all other dimensions including organisational and financial strengths, marketing abilities, relationship strength and logistics capabilities. This highlights that a retailer's technological capabilities can simplify and enhance the speed and flexibility of CPFR. These findings are in line with Lin et al (2004) who pointed out that appropriate IT infrastructure is a critical factor for adopting CPFR, and Fu et al. (2010) who found that a partner's information security system is a critical factor for adopting. In addition, Andrews (2008) and Büyüközkan and Vardaloğlu (2012) suggest that companies prefer to select partners who are compatible technological with themselves. However, it is well documented in the CPFR literature that technology can play a role as a critical enabler (as echoed in this study), it is worth mentioning that, technology is no longer seen as a major barrier to success (Europe ECR, 2001; Attaran and Attaran, 2007; Panahifar et al. 2014). This, example, clearly differentiates between the two different kinds of critical factors for CPFR implementation, enablers and barriers. To summarise, for successful CPFR implementation, technology is an important enabler however, it is also not a dominant barrier. 
In this study, $\mathrm{D}_{1}$ (organisational and financial strengths) and $\mathrm{D}_{2}$ (marketing abilities) are the two most important dimensions with the greatest collective Dimension Significance (DS) values. However, by further considering causal relationships, improving both marketing abilities and organisational and financial strengths dimensions cannot effectively strengthen retailers' performance in CPFR implementation alone because these two dimensions are extremely influenced by the other three dimensions.

\section{Conclusion}

Through a comprehensive literature review and expert opinion, the main retailer selection and evaluation factors for CPFR implementation are identified. A Fuzzy DEMATEL approach is then used to rank and analysis the interaction among identified criteria. The findings are finally evaluated using a case study from a high-tech industry. These criteria are categorised and quantified into two categories, cause and effect, with the complex interactions between retailer selection criteria transformed into a visible structural model, which captures and physically represents the most important and dominant factors. From a CPFR implementation theoretical perspective, this research significantly extends existing studies in this domain with 19 of the identified 24 criteria having not been previously identified and studied in the CPFR partnering domain.

From a practical based perspective, the presented model provides guidance in the preparation for and development of CPFR implementation projects for both manufacturers and retailers. The new evaluation model helps enhance manufacturers understanding of the relevant criteria and their attributes, providing significance and weightings for retailer identification and selection. It is also important that organisations understand the differences between cause and effect criteria, as identified visually in Figure 2. From a change perspective, the cause criteria are more readily adapted as direct input change will lead to direct output change. The effect criteria are more difficult to influence directly and identified change activities may not result in predicted better performance. This is due to the natural influence of the cause criteria on the effect criteria. Effect criteria are by their nature directly influenced by the many cause criteria and thus less predictable in change efforts. Thus, change efforts from a practice perspective are best spent on cause criteria, which if carried out correctly, should also positively impact upon effect criteria.

Of the 24 practical factors, a manufacturer's familiarity with the retailer has been shown to be the highest impact criteria in the study. As a cause criterion, this clearly suggests its practical importance and its relevant potential influence on the effect criteria. As is well documented in the literature, familiarity with trading partners can lead to a development of trust, which has been shown in previous studies to be an important dimension for trading partners. In other words, familiarity with the retailer allows the manufacturer to expedite CPFR implementation with them. This can also help a manufacturer in assessing the retailers' potential capabilities according to anticipated and realistic benefits, common business goals, and organisational and cultural differences. In terms of Rank of Importance (ROI), it is noted that a retailer's customer service orientation and capability $\left(\mathrm{C}_{12}\right)$ is the most important factor for CPFR implementation. Customer service capabilities like 'on-shelf availability' generate a better service to end users and require high level of manufacturer and retailer collaboration to succeed. Appropriate customer service capabilities enhance information visibility across partners which CPFR is essentially designed for. Additionally, in a broader sense, as this research considers comprehensive factors of the retailer selection process, the proposed evaluation structural model in supply chain management can also be used as an analytical monitoring tool to further develop an overall retailer evaluation approach. 
A limitation to the current study is its focus on the high-tech sector. The criteria developed in this study have been derived from a general literature review, but have been refined through an expert group with a primary focus in the high tech industries. Further, evaluation of these criteria in extended industrial domains would be useful to ensure broader validity. A second limitation is related to the validation of the findings. Although, an evaluation process has been conducted to evaluate the veracity of the research findings, extended industrial validation and testing of the developed model in an actual CPFR implementation project would significantly add to result validation. 


\section{References:}

Andrews, J. 2008. "CPFR: Considering the options, advantages and pitfalls." Supply and Demand Executive. 812.

Attaran, M., and Attaran, S. 2007. "Collaborative supply chain management: the most promising practice for building efficient and sustainable supply chains.” Business Process Management Journal 13 (3): 390-404.

Büyüközkan, G., \& Vardaloğlu, Z. 2012. "Analyzing of CPFR success factors using fuzzy cognitive maps in retail industry.” Expert Systems with Applications 39(12): 10438-10455.

Byrne, P.J. and Heavey, C. 2006. "The impact of information sharing and forecasting in capacitated industrial supply chains: A case study.” International Journal of Production Engineering 103 (1): 420-437.

Cavusgil, S.T., Yeoh, P. and Mitri, M. 1995. "Selecting foreign distributors-an expert systems approach", Industrial Marketing Management. 24: 297-304.

Chang, B., Chang, C. W., \& Wu, C. H. 2011. "Fuzzy DEMATEL method for developing supplier selection criteria." Expert Systems with Applications 38(3): 1850-1858.

Chung, W.C and Leung, S.W. 2005. "Collaborative planning, forecasting and replenishment: a case study in copper clad laminate industry." Production Planning and Control: The Management of Operations 16 (6): 563-574.

Daugherty, P. J., Richey, R. G., Roath, A. S., Min, S., Chen, H., Arndt, A. D., \& Genchev, S. E. (2006). Is collaboration paying off for firms?. Business Horizons, 49(1), 61-70.

Delbecq, A., A. Van de Ven, and D. Gustafson. 1975. Group Techniques for Program Planning. Glenview, IL: Scott, Foresman and Company.

Europe, E. C. R., \& Kronberg Accenture GmbH. 2001. A Guide to CPFR implementation. ECR Europe.

Fu, H.P., Chu, K.K., Lin, S.W., Chen, C.R. 2010. "A study on factors for retailers implementing CPFR - a fuzzy AHP analysis”, Journal of Systems Science and Systems Engineering 19 (2): 192-209.

Holmström, J., Främling, K., Kaipia, R., \& Saranen, J. (2002). Collaborative planning forecasting and replenishment: new solutions needed for mass collaboration. Supply Chain Management: An International Journal, 7(3), 136-145.

Huang, E. Y., \& Lin, S. C. (2006). How R\&D management practice affects innovation performance: An investigation of the high-tech industry in Taiwan. Industrial Management \& Data Systems, 106(7), 966-996.

Huang, S., Keskar, H. 2007. “Comprehensive and configurable metrics for supplier selection.” International Journal of Production Economics 105 (2): 510-523.

Ireland, R. D., Hitt, M. A., and Vaidyanath, D. 2002. "Alliance management as a source of competitive advantage." Journal of management 28 (3): 413-446.

Kelly, M.J., Schaan, J.-L., Joncas, H.2002. "Managing alliance relationships: key challenges in the early stages of Collaboration." R\&D Management 32 (1):11-22.

Lin, C. J., \& Wu, W. W. (2008). A causal analytical method for group decision-making under fuzzy environment. Expert Systems with Applications, 34(1), 205-213.

Lin, J. S. C., \& Chen, C. R. 2008. Determinants of manufacturers' selection of distributors. Supply Chain Management: An International Journal 13(5): 356-365. 
Lin, J.T., Yang, C.H and Lin, T.M. 2004. "A CPFR implementation methodology study - A Carpenter Mechanical Industry Case Study.” International Journal of Electronic Business Management 2 (3): 172-178.

Liou, J. J. 2012. "Developing an integrated model for the selection of strategic alliance partners in the airline industry." Knowledge-Based Systems 28: 59-67.

Liou, J. J., \& Chuang, Y. T. 2010. "Developing a hybrid multi-criteria model for selection of outsourcing providers.” Expert Systems with Applications 37 (5): 3755-3761.

Min, S., Roath, A. S., Daugherty, P. J., Genchev, S. E., Chen, H., Arndt, A. D., and Richey, R. G. 2005. "Supply chain collaboration: what's happening?” International Journal of Logistics Management 16 (2): 237-256.

Panahifar, F., Byrne, P. J., \& Heavey, C. 2014. “ISM analysis of CPFR implementation barriers.” International Journal of Production Research 52 (18): 5255-5272.

Panahifar, F., Byrne, P. J., \& Heavey, C. 2015b. "A hybrid approach to the study of CPFR implementation enablers." Production Planning \& Control, (ahead-of-print), 1-20.

Panahifar, F., Heavey, C., Byrne, P.J. 2015c. "A framework for Collaborative Planning, Forecasting and Replenishment (CPFR): State of the Art." Journal of Enterprise Information Management, Accepted for publication.

Panahifar, F., Heavey, C., Byrne, P.J. and Asif Salam, M. 2015a. "Partner Selection Factors for Successful CPFR Implementation Using Fuzzy DEMATEL.” Journal Of Economics, Business And Management, 3, 12, pp.1138-1145

Potter, M., Gordon, S., \& Hamer, P. (2004). The nominal group technique: a useful consensus methodology in physiotherapy research. New Zealand Journal of Physiotherapy, 32, 126-130.

Sahay, B. S. 2003. "Supply chain collaboration: the key to value creation.” Work Study 52(2), 76-83.

Sheffi, Y. 2002. “The value of CPFR”, RIRL Conference Proceedings, Lisbon. Portugal

Thomé, A. M. T., \& Hollmann, R. L. 2014. "Research synthesis in collaborative planning forecast and replenishment." Industrial Management \& Data Systems, 114(6), 7-7.

Tseng, M. L., \& Lin, Y. H. (2009). Application of Fuzzy DEMATEL to develop a cause and effect model of municipal solid waste management in Metro Manila. Environmental monitoring and assessment, 158(1-4), 519533.

VICS. 2013. Collaborative Planning, Forecasting \& Replenishment. Voluntary Interindustry Commerce Standards (VICS) Association. Accessed July 2013. http://www.vics.org/docs/committees/CPFR_Whitepaper_Spring_2008-VICS.pdf

Viswanathan, S., Widiarta, H., \& Piplani, R. 2007. Value of information exchange and synchronization in a multi-tier supply chain. International journal of production research 45 (21): 5057-5074.

Washida, Y. (2005). Collaborative structure between Japanese high-tech manufacturers and consumers. Journal of Consumer Marketing, 22(1), 25-34.

Wu, S. D., Erkoc, M., \& Karabuk, S. (2005). Managing capacity in the high-tech industry: A review of literature. The Engineering Economist, 50(2), 125-158.

Yuan, X., Shen, L., \& Ashayeri, J. (2010). Dynamic simulation assessment of collaboration strategies to manage demand gap in high-tech product diffusion. Robotics and Computer-Integrated Manufacturing, 26(6), 647657. 
Appendix A1. Assessment Data Fuzzy Matrix of an expert.

\begin{tabular}{|c|c|c|c|c|c|c|c|c|c|c|c|c|c|c|c|c|c|c|c|c|c|c|c|c|}
\hline 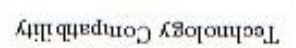 & 을 & 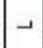 & 일 & $I$ & 을 & $I$ & ? & 울 & $\frac{9}{2}$ & 을 & 을 & 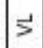 & 일 & $\mathrm{g}$ & 일 & ? & $\frac{9}{2}$ & - & $\mathrm{O}$ & $\mathrm{O}$ & $\frac{9}{2}$ & ? & 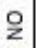 & \\
\hline 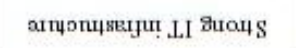 & 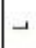 & \lrcorner & $\frac{9}{2}$ & $I$ & $\frac{9}{2}$ & I & 일 & 2 & $\frac{9}{2}$ & $\frac{9}{2}$ & 을 & $>$ & $\mathrm{g}$ & 2 & 일 & $\frac{2}{2}$ & 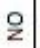 & - & 울 & $\frac{9}{2}$ & ? & @ & . & \pm \\
\hline 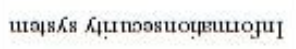 & 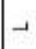 & - & $I$ & $I$ & $\frac{9}{2}$ & I & 일 & 울 & 울 & $\frac{9}{2}$ & $\frac{9}{2}$ & s & 인 & 인 & 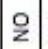 & 울 & 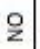 & $\rightarrow$ & 울 & $\frac{9}{2}$ & 인 & & $\stackrel{x}{5}$ & - \\
\hline 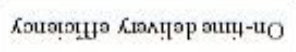 & I & I & $I$ & ـ & I & $I$ & s & $\frac{9}{2}$ & 을 & $\frac{9}{2}$ & $\frac{0}{2}$ & I & $\frac{9}{2}$ & - & \pm & $x$ & $\mathrm{O}$ & 5 & $\rightarrow$ & $\mathrm{I}$ & . & $\mathrm{g}$ & I & I \\
\hline 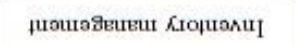 & I & $I$ & 일 & $I$ & I & $I$ & $\rightarrow$ & $\frac{9}{2}$ & 을 & $\frac{9}{2}$ & \$ & - & 高 & - & \pm & \pm & 인 & - & 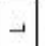 & . & 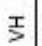 & ? & $\frac{1}{5}$ & - \\
\hline 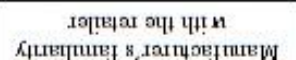 & 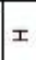 & $I$ & 올 & $I$ & I & - & 일 & 올 & $I$ & - & 울 & $I$ & 5 & 일 & 일 & $\stackrel{T}{>}$ & \lrcorner & $I$ & ' & 인 & ? & ? & ? & 인 \\
\hline นоب̣EIoqeпn & $I$ & 울 & 일 & $I$ & I & - & 일 & $x$ & $I$ & 을 & 울 & $I$ & $>$ & 일 & \pm & I & \pm & . & $I$ & ? & $>$ & ? & 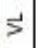 & $\Rightarrow$ \\
\hline 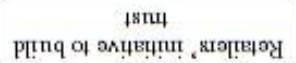 & $\stackrel{T}{>}$ & \lrcorner & 2 & - & $I$ & 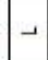 & ? & $I$ & $I$ & 을 & 2 & $I$ & 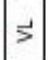 & 2 & \pm & $\stackrel{T}{>}$ & ' & $\frac{T}{3}$ & I & \lrcorner & 5 & \lrcorner & \lrcorner & - \\
\hline 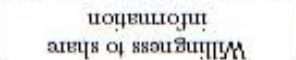 & $I$ & $\frac{9}{2}$ & \pm & - & I & $I$ & 2 & $I$ & 2 & 을 & 운 & \pm & $\rightarrow$ & 2 & - & ' & $\stackrel{T}{>}$ & $I$ & I & \lrcorner & ? & \pm & $\rightarrow$ & - \\
\hline 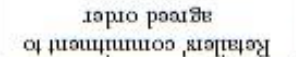 & 을 & 울 & ? & - & - & $I$ & ? & $I$ & $I$ & 을 & $\frac{9}{2}$ & - & $\$$ & 5 & . & I & $\rightarrow$ & \pm & $I$ & 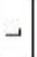 & I & ? & ? & 일 \\
\hline пвमоо & I & 을 & 일 & $\frac{9}{2}$ & \$ & $I$ & $\rightarrow$ & I & I & I & $I$ & I & \pm & . 1 & 일 & 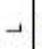 & $\frac{1}{2}$ & - & $\frac{9}{2}$ & I & 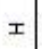 & ? & 일 & 일 \\
\hline 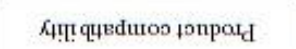 & $\frac{9}{2}$ & 일 & 일 & $I$ & ـ & - & 일 & $\frac{9}{2}$ & $\frac{9}{2}$ & \% & $I$ & इ & ' & $\frac{\mathrm{g}}{\mathrm{z}}$ & $\frac{9}{2}$ & 울 & \% & $\frac{9}{2}$ & $\frac{9}{2}$ & @ & २ & $\frac{\mathrm{O}}{2}$ & $\frac{1}{2}$ & $\frac{1}{2}$ \\
\hline 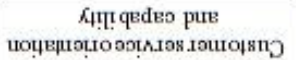 & $I$ & 일 & I & $I$ & I & $I$ & $\Rightarrow$ & $I$ & $I$ & I & $I$ & . & - & \pm & \pm & $\stackrel{T}{>}$ & - & \pm & 울 & 2 & \pm & 인 & 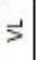 & 인 \\
\hline 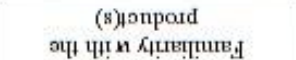 & 은 & $I$ & $I$ & $I$ & I & 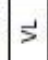 & \% & 5 & - & $I$ & 1 & \pm & \pm & 인 & 인 & I & 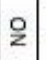 & $\rightarrow$ & 2 & ? & ? & २ & 2 & 2 \\
\hline 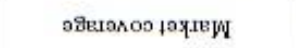 & $I$ & \pm & $I$ & $\frac{1}{3}$ & I & I & 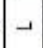 & $\stackrel{x}{>}$ & $\frac{1}{3}$ & ' & $I$ & $I$ & $\$$ & I & 일 & I & 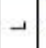 & \pm & 2 & \pm & 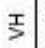 & ? & 일 & 일 \\
\hline 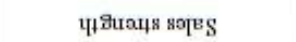 & I & $I$ & $\stackrel{T}{>}$ & I & I & I & ـ & I & . & $\stackrel{T}{>}$ & I & I & $\$$ & I & \pm & I & $\lessgtr$ & \pm & 일 & I & $\stackrel{\Gamma}{\lessgtr}$ & ـ & $\rightarrow$ & 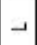 \\
\hline 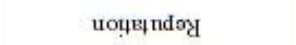 & $I$ & \pm & \pm & I & $\stackrel{I}{>}$ & $I$ & 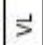 & . & I & I & $I$ & \pm & $\$$ & I & \% & \pm & 일 & \pm & $\frac{9}{2}$ & I & $\stackrel{\Upsilon}{\supset}$ & ــ & 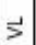 & 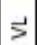 \\
\hline 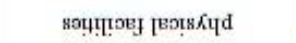 & - & $\frac{2}{2}$ & - & I & 올 & I & . & \$ & I & ـ & 을 & 일 & 号 & 5 & 일 & $\mathrm{q}$ & 인 & 일 & 울 & 일 & 을 & ? & 일 & 일 \\
\hline 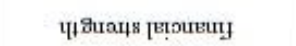 & $I$ & $\rightarrow$ & $I$ & $I$ & I & . & s & ـ & I & I & - & $I$ & $\$$ & \pm & \pm & \pm & इ & $\rightarrow$ & : & \pm & I & ـ & $\rightarrow$ & ـ \\
\hline 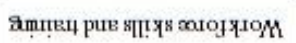 & $\stackrel{I}{>}$ & I & I & $I$ & , & I & 일 & 을 & 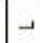 & $\frac{9}{2}$ & 울 & I & 일 & 일 & 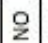 & I & 일 & 일 & $\frac{9}{2}$ & 일 & 울 & 일 & \lrcorner & 일 \\
\hline 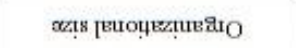 & 을 & 일 & \pm & , & I & I & 일 & 올 & I & $I$ & 을 & 일 & 일 & \pm & 2 & 울 & 일 & 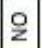 & 울 & ? & 울 & 인 & 인 & 일 \\
\hline 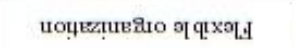 & I & $\stackrel{I}{>}$ & . & $I$ & I & 인 & 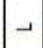 & 을 & 울 & 을 & I & $I$ & 일 & 일 & 일 & I & 일 & - & 울 & 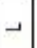 & ? & I & I & I \\
\hline 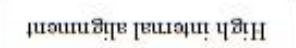 & $\stackrel{I}{>}$ & ' & $I$ & I & I & ら & \pm & ــ & $\frac{0}{2}$ & 을 & - & 을 & $I$ & $\frac{1}{2}$ & 일 & $\stackrel{I}{>}$ & 일 & $\stackrel{7}{>}$ & 일 & 일 & 일 & 일 & $I$ & ــ \\
\hline 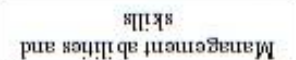 & . & 을 & 2 & 을 & I & $\$$ & $\$$ & 을 & 을 & 을 & $\stackrel{I}{\supset}$ & $\stackrel{T}{\lessgtr}$ & \pm & 2 & 인 & I & \% & - & $\frac{9}{2}$ & \% & ? & \% & \pm & 2 \\
\hline & 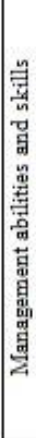 & 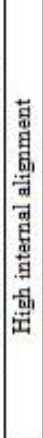 & 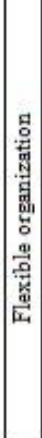 & 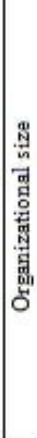 & 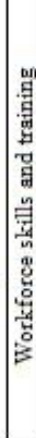 & 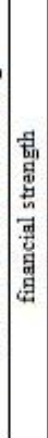 & 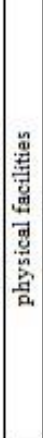 & 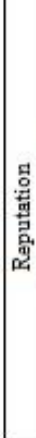 & 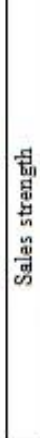 & 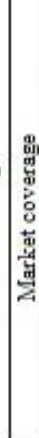 & 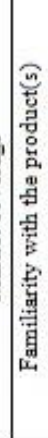 & 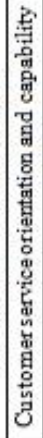 & 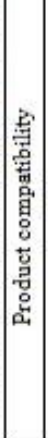 & 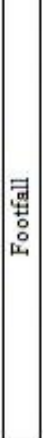 & 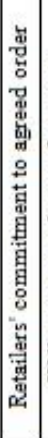 & 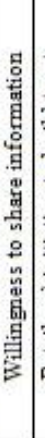 & 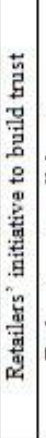 & 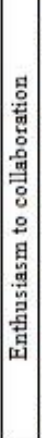 & 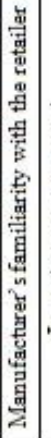 & 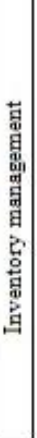 & 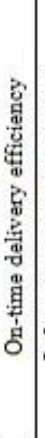 & 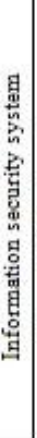 & 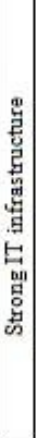 & 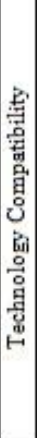 \\
\hline
\end{tabular}


Appendix A2. The Initial direct-relation fuzzy matrix $\tilde{Z}$.

\begin{tabular}{|c|c|c|c|c|c|c|c|c|c|c|c|c|c|c|c|c|c|c|c|c|c|c|c|c|}
\hline & 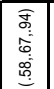 & 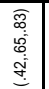 & 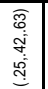 & 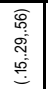 & 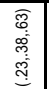 & 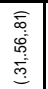 & 然 & 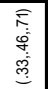 & |ct & 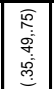 & |ch & 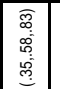 & 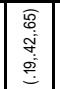 & 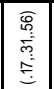 & 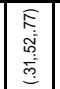 & 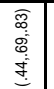 & 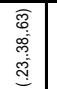 & 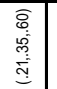 & 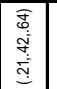 & 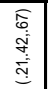 & 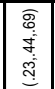 & 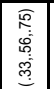 & 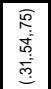 & \begin{tabular}{|l}
$\hat{0}$ \\
$\dot{0}$ \\
$\dot{0}$
\end{tabular} \\
\hline & 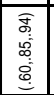 & 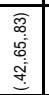 & 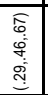 & 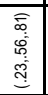 & 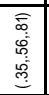 & 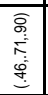 & 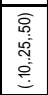 & 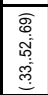 & 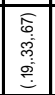 & 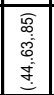 & 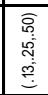 & 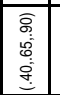 & 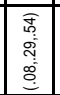 & 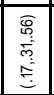 & 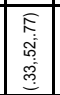 & 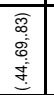 & 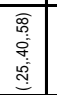 & 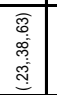 & 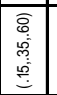 & 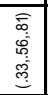 & 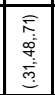 & 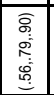 & 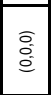 & 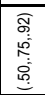 \\
\hline & 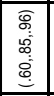 & 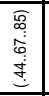 & 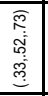 & 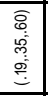 & 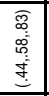 & 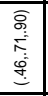 & 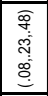 & 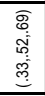 & 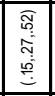 & 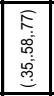 & 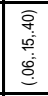 & 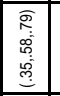 & 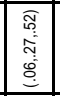 & 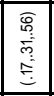 & 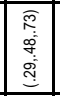 & $\overline{\underline{g}}$ & 苞 & 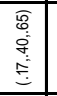 & 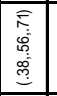 & 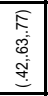 & 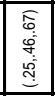 & 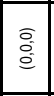 & 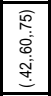 & 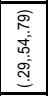 \\
\hline$N$ & 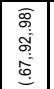 & 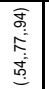 & 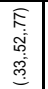 & 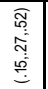 & 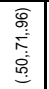 & 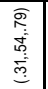 & 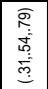 & 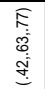 & 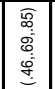 & 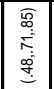 & 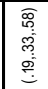 & 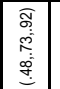 & 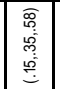 & 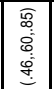 & 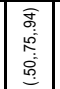 & 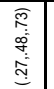 & 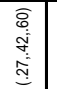 & 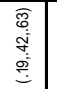 & 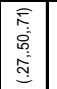 & 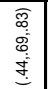 & $\begin{array}{l}\hat{0} \\
\dot{0} \\
\dot{0}\end{array}$ & 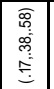 & 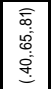 & 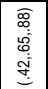 \\
\hline$\overline{\mathrm{N}}$ & 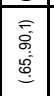 & 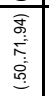 & 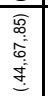 & 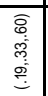 & 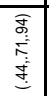 & 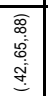 & 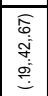 & $\bar{E}$ & త্ & 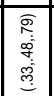 & 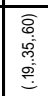 & 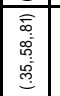 & 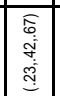 & 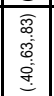 & 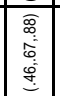 & 高 & 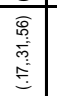 & 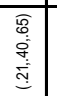 & 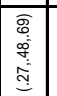 & 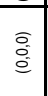 & 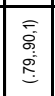 & 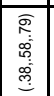 & 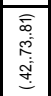 & $E$ \\
\hline$=$ & 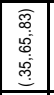 & 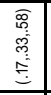 & 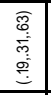 & 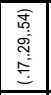 & 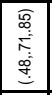 & 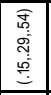 & 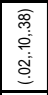 & 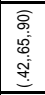 & 产 & 5 & 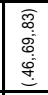 & 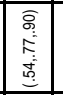 & 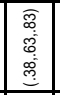 & \begin{tabular}{|l|}
\multirow{6}{\sigma}{} \\
\end{tabular} & $\widehat{\widehat{8}}$ & $\begin{array}{l}\mathbb{E} \\
\mathbb{F}_{\infty}\end{array}$ & 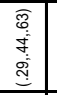 & 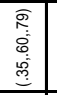 & \begin{tabular}{|l} 
\\
o. \\
$\dot{0}$
\end{tabular} & 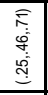 & 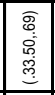 & 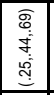 & 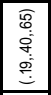 & 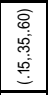 \\
\hline$\therefore$ & 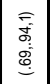 & 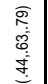 & 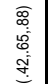 & 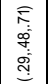 & 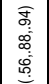 & 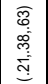 & 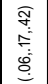 & 鹿 & 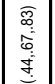 & 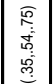 & 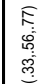 & $\begin{array}{l}\text { 嵩 } \\
\text { o }\end{array}$ & 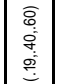 & 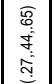 & ले & $\begin{array}{l}0 \\
0 \\
0 \\
0 \\
0 \\
0\end{array}$ & 它 & \begin{tabular}{|l|}
$\hat{0}$ \\
$\dot{0}$ \\
$\dot{s}$
\end{tabular} & 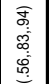 & 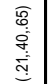 & 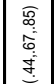 & 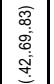 & 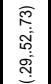 & 高 \\
\hline $\bar{\vdots}$ & 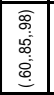 & 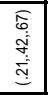 & 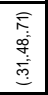 & 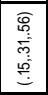 & 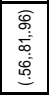 & 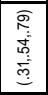 & 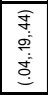 & $\stackrel{8}{\circ}$ & 可 & 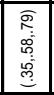 & 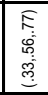 & & $\widehat{\widehat{\sigma}}$ & & & & & 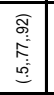 & $\overline{\mathrm{o}}$ & $\widehat{\mathscr{8}}$ & $\widehat{\widehat{\sigma}}$ & 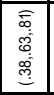 & 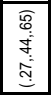 & $\overline{\bar{\Phi}}$ \\
\hline$\div$ & 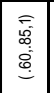 & 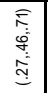 & 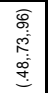 & 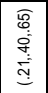 & 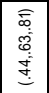 & 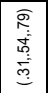 & 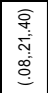 & \begin{tabular}{l}
$\bar{\Phi}$ \\
$:$ \\
\hdashline
\end{tabular} & 窎 & 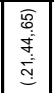 & $\sqrt{8}$ & & & & & 它 & (1) & $\bar{\infty}$ & & & @ & 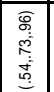 & 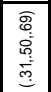 & 9 \\
\hline 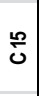 & 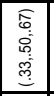 & 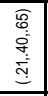 & 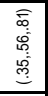 & 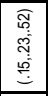 & 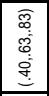 & 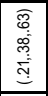 & 勇 & 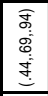 & 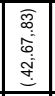 & $\overline{\mathrm{\Phi}}$ & $\widehat{\widehat{s}}$ & $\widehat{\overline{\mathrm{g}}}$ & 舀 & & 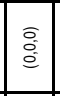 & $\widehat{\underline{\alpha}}$ & & \begin{tabular}{|c|}
$\Phi$ \\
\end{tabular} & & \begin{tabular}{|l|}
$\widehat{5}$ \\
0 \\
0
\end{tabular} & 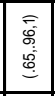 & 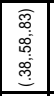 & 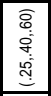 & 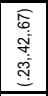 \\
\hline 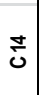 & 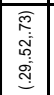 & 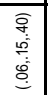 & 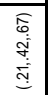 & 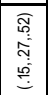 & 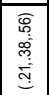 & 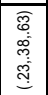 & 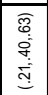 & & 1 & 芯 & $\begin{array}{l}\mathbb{E} \\
\text { E. } \\
\text { s. }\end{array}$ & $\widehat{\bar{\infty}}$ & $\widehat{\Phi}$ & o. & & +1 & & 总 & & צִ & $\widehat{\widetilde{\sigma}}$ & 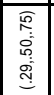 & 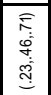 & ⿸厂 \\
\hline m & 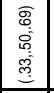 & 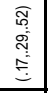 & 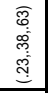 & 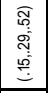 & 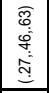 & 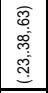 & $\underline{\Phi}$ & $=$ & $\bar{a}$ & 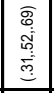 & $\begin{array}{l}\text { 秀 } \\
\text { s. }\end{array}$ & $\widehat{\bar{ळ}}$ & 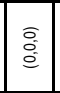 & $\overline{\widetilde{ø}}$ & & & & & & & & 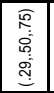 & |ct & $\overline{\widehat{\overparen{6}}}$ \\
\hline 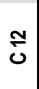 & 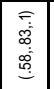 & 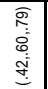 & 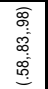 & 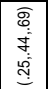 & 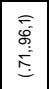 & 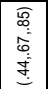 & $\overline{0}$ & مُ & 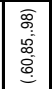 & 势 & : & 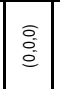 & స్త్ర & & & f & $\widehat{\Phi}$ & \begin{tabular}{|c|}
5 \\
\end{tabular} & & & 兽 & & 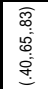 & $\overline{8}$ \\
\hline- & 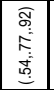 & 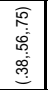 & 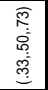 & 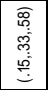 & 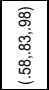 & 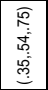 & $\overline{\sigma o}$ & $\widehat{\widetilde{\infty}}$ & & $\begin{array}{ll}\widehat{\bar{g}} \\
\text { os }\end{array}$ & $\begin{array}{l}\dot{\theta}_{0} \\
\dot{0}\end{array}$ & & $\overline{8}$ & & & & & 象 & & & & & 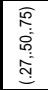 & 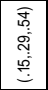 \\
\hline & 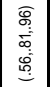 & 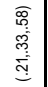 & 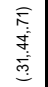 & 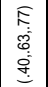 & 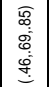 & 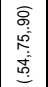 & 嘿 & & & $\begin{array}{l}8 \\
\vdots \\
0 \\
\vdots\end{array}$ & 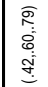 & & & & & & & & & & & & 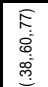 & $\overline{\mathrm{\sigma}}$ \\
\hline & 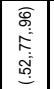 & 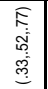 & 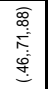 & 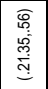 & 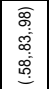 & 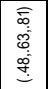 & 焉 & $\bar{E}$ & $\begin{array}{c}\bar{a} \\
\dot{a} \\
\dot{0}\end{array}$ & 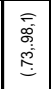 & 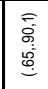 & & fo & & & & 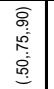 & $\stackrel{\infty}{\infty}$ & & & 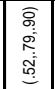 & क़ุ & 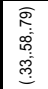 & త్ \\
\hline ¿ & 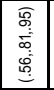 & 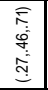 & 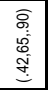 & 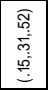 & 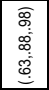 & 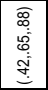 & $\frac{6}{6}$ & 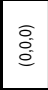 & & & & & & & & & & & & & & & & 哀 \\
\hline ؛ & 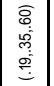 & 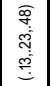 & 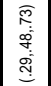 & 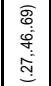 & 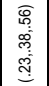 & 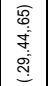 & $\begin{array}{l}\bar{a} \\
\dot{a} \\
\dot{a}\end{array}$ & ్ָత & 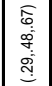 & 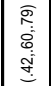 & 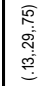 & & & & & तु & స్త్ & $\widehat{\underline{x}}$ & ã & $\tilde{\cong}$ & | & | & & $\overline{\overline{6}}$ \\
\hline 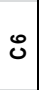 & 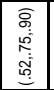 & 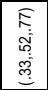 & 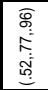 & 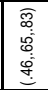 & 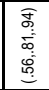 & 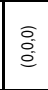 & & & 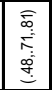 & & & & & & & & & & & ge & 要 & 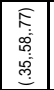 & 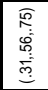 & 象 \\
\hline తै & 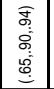 & 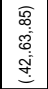 & 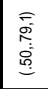 & 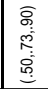 & 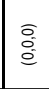 & 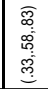 & & & & & 可 & & & & & & & & & $\stackrel{\infty}{g}$ & & & & बิ \\
\hline J & 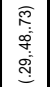 & 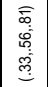 & 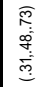 & $\therefore$ & 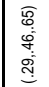 & 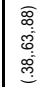 & & & & f & $\ddot{\simeq}$ & & & & & & & & बึ่ & $\overline{6}$ & $\mid$ & 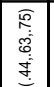 & & $\overline{\text { S }}$ \\
\hline & 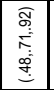 & 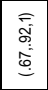 & $\begin{array}{c}\bar{\delta} \\
\bar{\delta} \\
\dot{0}\end{array}$ & 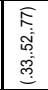 & 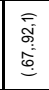 & 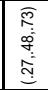 & 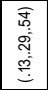 & $\overline{8}$ & $E$ & & 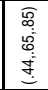 & & & & & & $\mid$ & $\stackrel{\infty}{\infty}$ & 禺 & 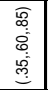 & 过 & \begin{tabular}{|l|}
$g$ \\
$g$ \\
$g$
\end{tabular} & 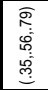 & $\widehat{\overline{6}}$ \\
\hline o & 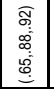 & $\therefore$ & 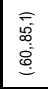 & 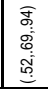 & 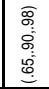 & 苞 & & & & & ç & & & & & & & & & 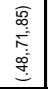 & 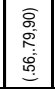 & 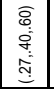 & 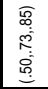 & 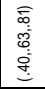 \\
\hline & 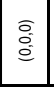 & 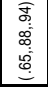 & 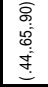 & 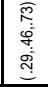 & $\begin{array}{l}\text { 䐱 } \\
\vdots \\
\vdots\end{array}$ & & & & & & ఫ్ & 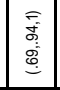 & & & א్ & 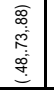 & 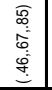 & & & 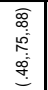 & & 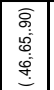 & 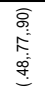 & דَّ \\
\hline
\end{tabular}

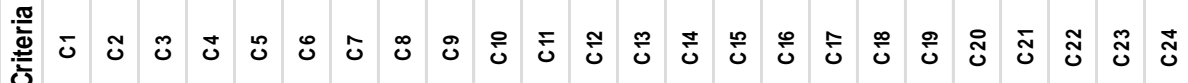


Appendix A3. The normalized initial direction-relation fuzzy matrix $\tilde{X}$.

\begin{tabular}{|c|c|c|c|c|c|c|c|c|c|c|c|c|c|c|c|c|c|c|c|c|c|c|c|c|}
\hline & 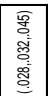 & 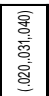 & 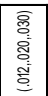 & 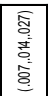 & 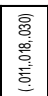 & 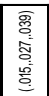 & 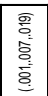 & 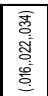 & 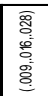 & 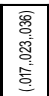 & 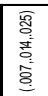 & 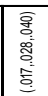 & 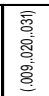 & 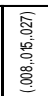 & 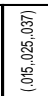 & 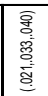 & 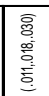 & 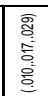 & 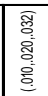 & 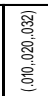 & 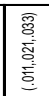 & 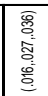 & 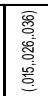 & बू. \\
\hline$\tilde{\varepsilon}$ & 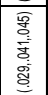 & 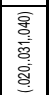 & 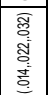 & 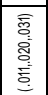 & 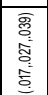 & 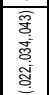 & 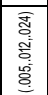 & 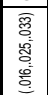 & 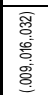 & 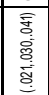 & 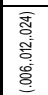 & 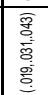 & 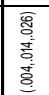 & 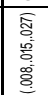 & 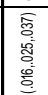 & 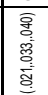 & 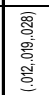 & 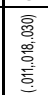 & 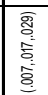 & 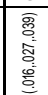 & 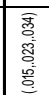 & 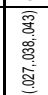 & | & \\
\hline 8 & 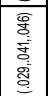 & 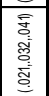 & 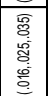 & 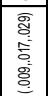 & 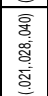 & 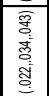 & 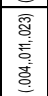 & 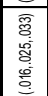 & 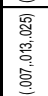 & 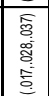 & 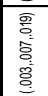 & 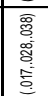 & 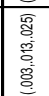 & 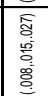 & 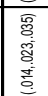 & 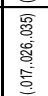 & 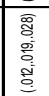 & 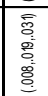 & 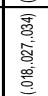 & 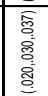 & 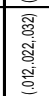 & 产 & 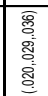 & \\
\hline ¿ & 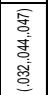 & 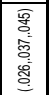 & 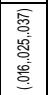 & 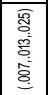 & 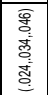 & 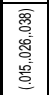 & 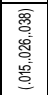 & 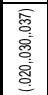 & 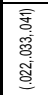 & 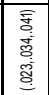 & 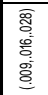 & 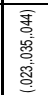 & 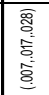 & 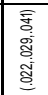 & 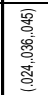 & 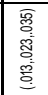 & 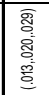 & 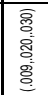 & 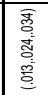 & 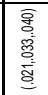 & 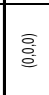 & 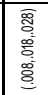 & 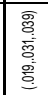 & \\
\hline ฮ & 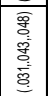 & 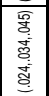 & 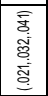 & 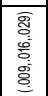 & 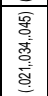 & 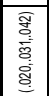 & 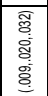 & 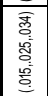 & 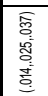 & 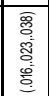 & 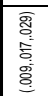 & 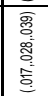 & 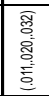 & 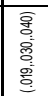 & 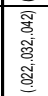 & 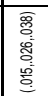 & 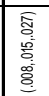 & 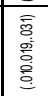 & 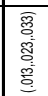 & | & 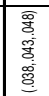 & 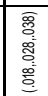 & 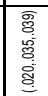 & 第 \\
\hline 운 & 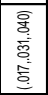 & 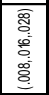 & 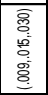 & 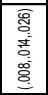 & 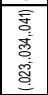 & 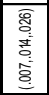 & 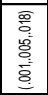 & 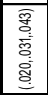 & 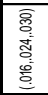 & 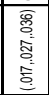 & 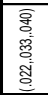 & 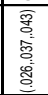 & 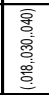 & 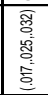 & 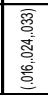 & 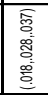 & 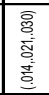 & 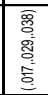 & $\mid$\begin{tabular}{|c|} 
\\
a
\end{tabular} & 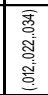 & 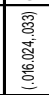 & 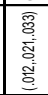 & 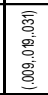 & \\
\hline $\bar{c}$ & 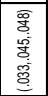 & 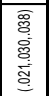 & 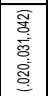 & 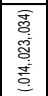 & 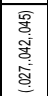 & 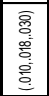 & 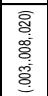 & 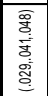 & 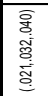 & 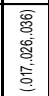 & 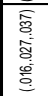 & 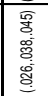 & 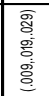 & 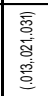 & 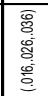 & 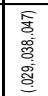 & 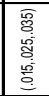 & 高 & 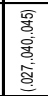 & 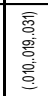 & 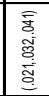 & 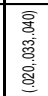 & 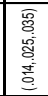 & \\
\hline 5 & 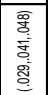 & 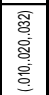 & 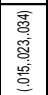 & 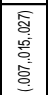 & 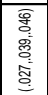 & 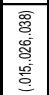 & \begin{tabular}{|l|}
$\overline{\bar{y}}$ \\
$\bar{s}$ \\
$\bar{s}$ \\
$\bar{s}$ \\
\end{tabular} & 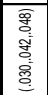 & 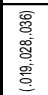 & 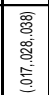 & 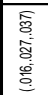 & 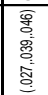 & 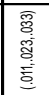 & 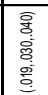 & 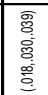 & 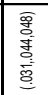 & 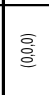 & 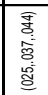 & 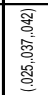 & 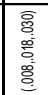 & 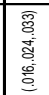 & 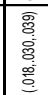 & 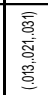 & \\
\hline$\frac{c}{c}$ & 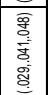 & 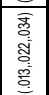 & 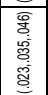 & 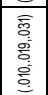 & 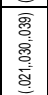 & 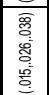 & 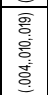 & 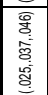 & 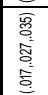 & 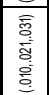 & 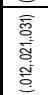 & 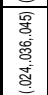 & 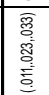 & 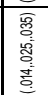 & 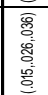 & 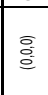 & 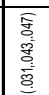 & 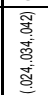 & 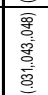 & 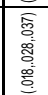 & 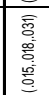 & 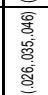 & 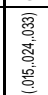 & $\overline{\bar{\rho}}$ \\
\hline है & 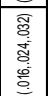 & 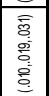 & 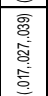 & 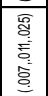 & 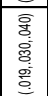 & 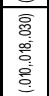 & 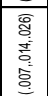 & 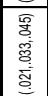 & 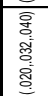 & 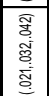 & 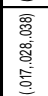 & 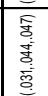 & 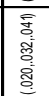 & 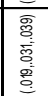 & 高 & 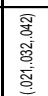 & 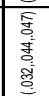 & 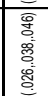 & 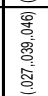 & 兽 & 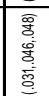 & 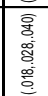 & 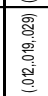 & \\
\hline$\stackrel{5}{0}$ & 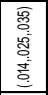 & 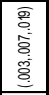 & 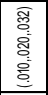 & 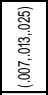 & 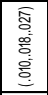 & 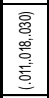 & 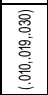 & 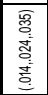 & 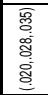 & 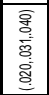 & 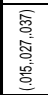 & 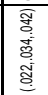 & 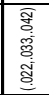 & $\begin{array}{l}\text { à } \\
\text { a }\end{array}$ & 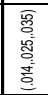 & 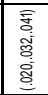 & 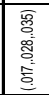 & 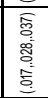 & 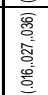 & $\overline{\text { g. }}$ & 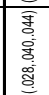 & 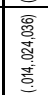 & 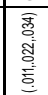 & \\
\hline 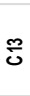 & 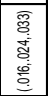 & 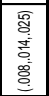 & 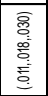 & 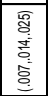 & 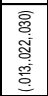 & 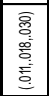 & 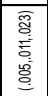 & 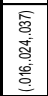 & 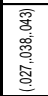 & 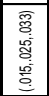 & 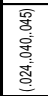 & 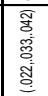 & 总 & 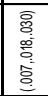 & 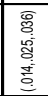 & 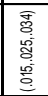 & 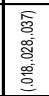 & 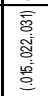 & 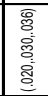 & 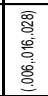 & 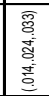 & 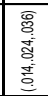 & 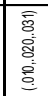 & \\
\hline ؟̃ & 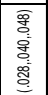 & 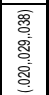 & 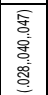 & 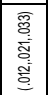 & 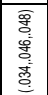 & 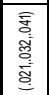 & 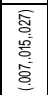 & 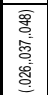 & 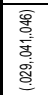 & 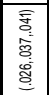 & 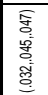 & & 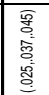 & 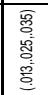 & 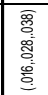 & 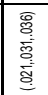 & 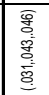 & 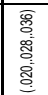 & 鸽 & $\overline{\mathrm{s}}$ & $\mid \overline{8}$ & 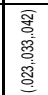 & 亨 & $\bar{\infty}$ \\
\hline$\overline{0}$ & 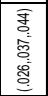 & 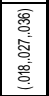 & 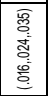 & 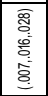 & 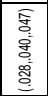 & 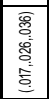 & 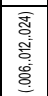 & 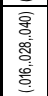 & 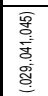 & 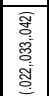 & 产 & 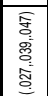 & 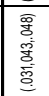 & 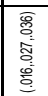 & 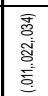 & 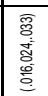 & 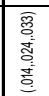 & 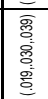 & 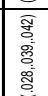 & \% & 产 & 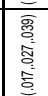 & \begin{tabular}{|l}
8 \\
8
\end{tabular} & 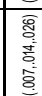 \\
\hline 인 & 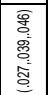 & 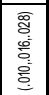 & 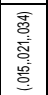 & 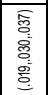 & 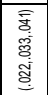 & 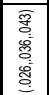 & 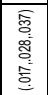 & 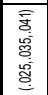 & 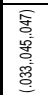 & 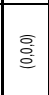 & 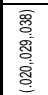 & 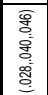 & 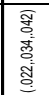 & 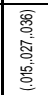 & 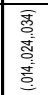 & 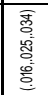 & 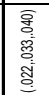 & 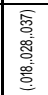 & 释 & 㠃 & 要 & 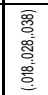 & 愈 & 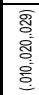 \\
\hline 8 & 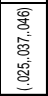 & 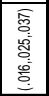 & 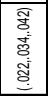 & 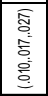 & 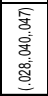 & 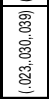 & 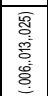 & \begin{tabular}{|l|} 
㩊 \\
\end{tabular} & 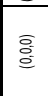 & 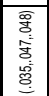 & 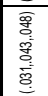 & 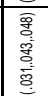 & 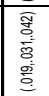 & 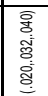 & 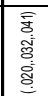 & 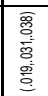 & 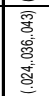 & 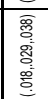 & 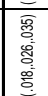 & $\overline{\bar{g}}$ & 䋧 & 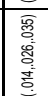 & 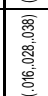 & 5 \\
\hline ॐ & 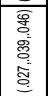 & 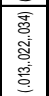 & 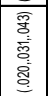 & 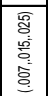 & 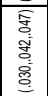 & 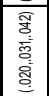 & 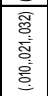 & 兽 & 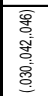 & 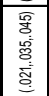 & 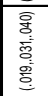 & 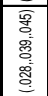 & 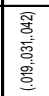 & 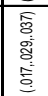 & 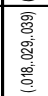 & 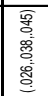 & 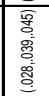 & 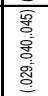 & 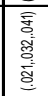 & 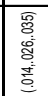 & 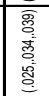 & 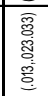 & 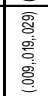 & 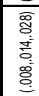 \\
\hline ¿ & 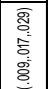 & 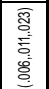 & 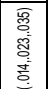 & 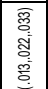 & 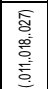 & 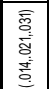 & \begin{tabular}{|l|l} 
\\
0 \\
0 \\
0
\end{tabular} & 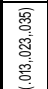 & $\bar{~} \bar{g}$ & 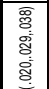 & 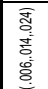 & 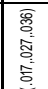 & 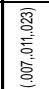 & 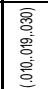 & 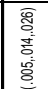 & 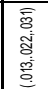 & 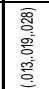 & 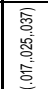 & 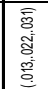 & 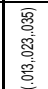 & 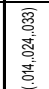 & 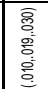 & 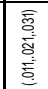 & 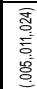 \\
\hline ¿ & 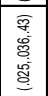 & 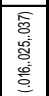 & 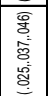 & 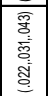 & 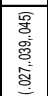 & $\begin{array}{l}\text { à } \\
\text { en }\end{array}$ & 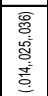 & 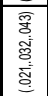 & 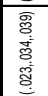 & 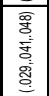 & 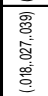 & 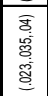 & 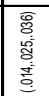 & 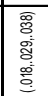 & 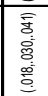 & 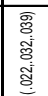 & 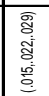 & 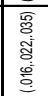 & 幽 & 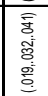 & 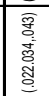 & 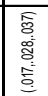 & 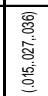 & 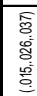 \\
\hline ن & 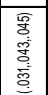 & 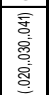 & 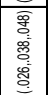 & 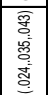 & (a & 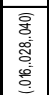 & 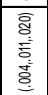 & 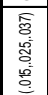 & 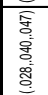 & 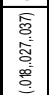 & 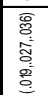 & 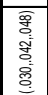 & 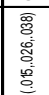 & 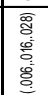 & 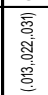 & 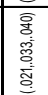 & 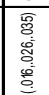 & 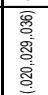 & 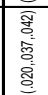 & 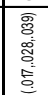 & 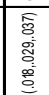 & 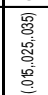 & 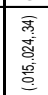 & \begin{tabular}{|c|c|} 
可 \\
\end{tabular} \\
\hline తే & 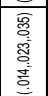 & 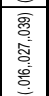 & 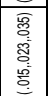 & 总 & 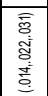 & 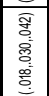 & 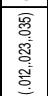 & 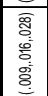 & 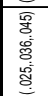 & 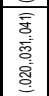 & 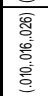 & 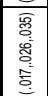 & 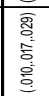 & 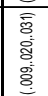 & 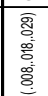 & 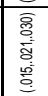 & 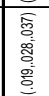 & 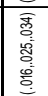 & 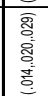 & 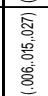 & 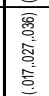 & 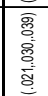 & 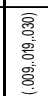 & 玄 \\
\hline ฮ & 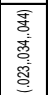 & 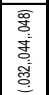 & 高 & 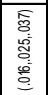 & 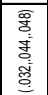 & 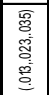 & \begin{tabular}{|l|l}
. \\
\end{tabular} & 要 & 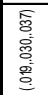 & कू & 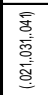 & 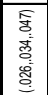 & 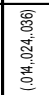 & 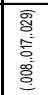 & 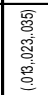 & 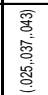 & 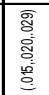 & 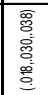 & 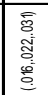 & 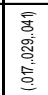 & 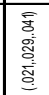 & 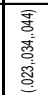 & 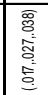 & $\overline{\bar{p}}$ \\
\hline తิ & 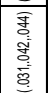 & : & 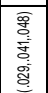 & 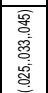 & 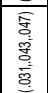 & \begin{tabular}{|l|} 
愛 \\
s. \\
s. \\
s. \\
\end{tabular} & 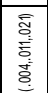 & 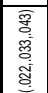 & 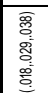 & $\overline{\mathrm{g}}$ & 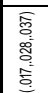 & 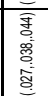 & 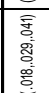 & 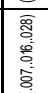 & 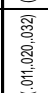 & 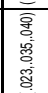 & 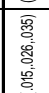 & 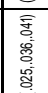 & 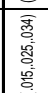 & 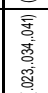 & 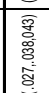 & 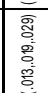 & 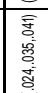 & 可 \\
\hline $\bar{\delta}$ & 高 & 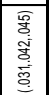 & 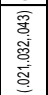 & 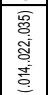 & 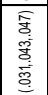 & 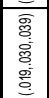 & 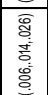 & 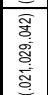 & 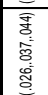 & 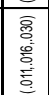 & 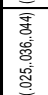 & 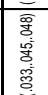 & 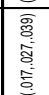 & 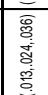 & 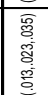 & 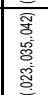 & 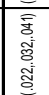 & 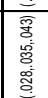 & 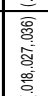 & 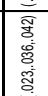 & 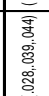 & 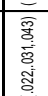 & 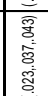 & \\
\hline & $\bar{c}$ & $\widetilde{\delta}$ & $\tilde{c}$ & J & 8 & 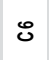 & $\hat{\jmath}$ & రొ & : & 0 & 0 & 0 & 0 & 0 & & 0 & & 0 & & $\delta$ & E & o & 0 & \\
\hline
\end{tabular}


Appendix A4. The values of $X_{l}, X_{m}$ and $X_{r}$.

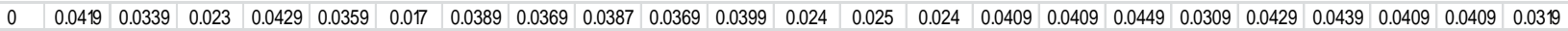

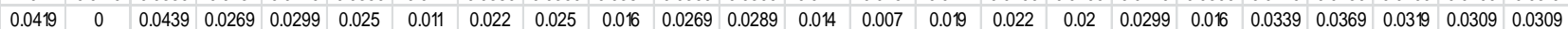
\begin{tabular}{l|l|l|l|l|l|l|l|l|l|l|l|l|l|l|l|l|l|l|l|l|l|l|l|l|l|l|l|l|l|l}
0.0319 & 0.0409 & 0 & 0.023 & 0.0379 & 0.0369 & 0.023 & 0.0309 & 0.0339 & 0.021 & 0.024 & 0.0399 & 0.018 & 0.02 & 0.0269 & 0.0349 & 0.023 & 0.0309 & 0.015 & 0.0319 & 0.025 & 0.025 & 0.022 & 0.02
\end{tabular}

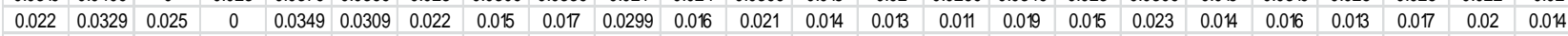
$\begin{array}{llllllllllllllllllllllllll}0.0429 & 0.0429 & 0.0439 & 0.022 & 0 & 0.0389 & 0.018 & 0.0419 & 0.0399 & 0.0329 & 0.0399 & 0.0459 & 0.022 & 0.018 & 0.0299 & 0.0299 & 0.0389 & 0.0419 & 0.0339 & 0.0339 & 0.0339 & 0.0279 & 0.0269 & 0.018\end{array}$

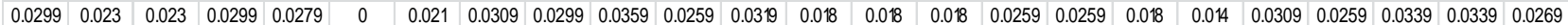

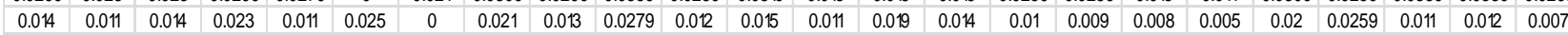
\begin{tabular}{ll|l|l|l|l|l|l|l|l|l|l|l|l|l|l|l|l|l|l|l|l|l|l|l|l|l|l|l|l|l}
0.0289 & 0.0329 & 0.0259 & 0.016 & 0.0248 & 0.0319 & 0.023 & 0 & 0.0369 & 0.0349 & 0.0279 & 0.0379 & 0.024 & 0.024 & 0.0329 & 0.0369 & 0.0419 & 0.0409 & 0.0309 & 0.025 & 0.0299 & 0.025 & 0.025 & 0.022 \\
\hline
\end{tabular} \begin{tabular}{ll|l|l|l|l|l|l|l|l|l|l|l|l|l|l|l|l|l|l|l|l|l|l|l}
0.0369 & 0.0289 & 0.0299 & 0.0359 & 0.0399 & 0.0339 & 0.023 & 0.0419 & 0 & 0.0449 & 0.0409 & 0.0409 & 0.0379 & 0.0279 & 0.0319 & 0.0269 & 0.0279 & 0.0319 & 0.024 & 0.025 & 0.0329 & 0.013 & 0.016 & 0.016
\end{tabular} $\begin{array}{llllllllllllllllllllllllllll}0.0164 & 0.018 & 0.019 & 0.0309 & 0.0269 & 0.0409 & 0.0289 & 0.0349 & 0.0469 & 0 & 0.0329 & 0.0369 & 0.025 & 0.0311 & 0.0319 & 0.021 & 0.0279 & 0.0259 & 0.0269 & 0.023 & 0.0339 & 0.0279 & 0.0299 & 0.0234\end{array}$

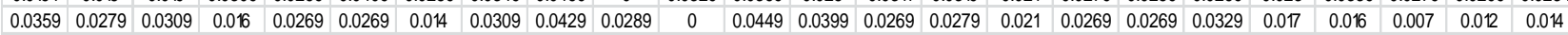
$\begin{array}{lllllllllllllllllllllllllll}0.0449 & 0.0379 & 0.0339 & 0.0259 & 0.0419 & 0.0349 & 0.0269 & 0.0389 & 0.0429 & 0.0399 & 0.0389 & 0 & 0.0329 & 0.0339 & 0.0439 & 0.0359 & 0.0389 & 0.0379 & 0.0369 & 0.0279 & 0.0349 & 0.0279 & 0.0309 & 0.0279\end{array}$

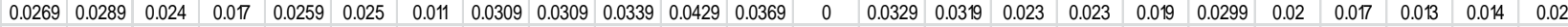
\begin{tabular}{lllllllllllllllllllllllllllll}
0.024 & 0.016 & 0.017 & 0.02 & 0.016 & 0.0289 & 0.019 & 0.0289 & 0.0319 & 0.0269 & 0.0269 & 0.025 & 0.018 & 0 & 0.0309 & 0.025 & 0.0299 & 0.021 & 0.025 & 0.0299 & 0.0289 & 0.015 & 0.015 & 0.015 \\
\hline
\end{tabular} $\begin{array}{llllllllllllllllllllllllllll}0.023 & 0.02 & 0.023 & 0.018 & 0.022 & 0.0299 & 0.014 & 0.0289 & 0.0319 & 0.024 & 0.022 & 0.0279 & 0.025 & 0.025 & 0 & 0.0259 & 0.0299 & 0.0259 & 0.024 & 0.0319 & 0.0359 & 0.023 & 0.025 & 0.025\end{array}$ $\begin{array}{lllllllllllllllllllllllll}0.0349 & 0.0349 & 0.0369 & 0.021 & 0.0329 & 0.0319 & 0.022 & 0.0379 & 0.0309 & 0.025 & 0.024 & 0.0309 & 0.025 & 0.0319 & 0.0319 & 0 & 0.0439 & 0.0379 & 0.0279 & 0.0259 & 0.023 & 0.0259 & 0.0329 & 0.0329\end{array}$ \begin{tabular}{llllllllllllllllllllllllll}
0.0319 & 0.0259 & 0.02 & 0.0279 & 0.0259 & 0.022 & 0.019 & 0.0389 & 0.0359 & 0.0329 & 0.024 & 0.0429 & 0.0279 & 0.0279 & 0.0439 & 0.0429 & 0 & 0.025 & 0.021 & 0.015 & 0.02 & 0.019 & 0.019 & 0.018 \\
\hline
\end{tabular} \begin{tabular}{|l|l|l|l|l|l|l|l|l|l|l|l|l|l|l|l|l|l|l|l|l|l|l|l|l}
0.035 & 0.0359 & 0.0299 & 0.025 & 0.0289 & 0.022 & 0.0269 & 0.0399 & 0.0289 & 0.0279 & 0.0299 & 0.0279 & 0.022 & 0.0279 & 0.0379 & 0.0339 & 0.0369 & 0 & 0.0289 & 0.019 & 0.02 & 0.019 & 0.018 & 0.017
\end{tabular} $\begin{array}{lllllllllllllllllllllllll}0.0269 & 0.025 & 0.022 & 0.02 & 0.0369 & 0.0339 & 0.022 & 0.0319 & 0.0259 & 0.0349 & 0.0389 & 0.0289 & 0.0299 & 0.0269 & 0.0389 & 0.0429 & 0.0369 & 0.0399 & 0 & 0.023 & 0.024 & 0.0269 & 0.017 & 0.02\end{array}$ \begin{tabular}{l|l|l|l|l|l|l|l|l|l|l|l|l|l|l|l|l|l|l|l|l|l|l|l|l|l}
0.0359 & 0.0339 & 0.0289 & 0.015 & 0.0279 & 0.0319 & 0.023 & 0.0259 & 0.021 & 0.025 & 0.024 & 0.021 & 0.016 & 0.023 & 0.0269 & 0.0279 & 0.018 & 0.019 & 0.022 & 0 & 0.0329 & 0.0299 & 0.0269 & 0.02 \\
\hline
\end{tabular} \begin{tabular}{ll|l|l|l|l|l|l|l|l|l|l|l|l|l|l|l|l|l|l|l|l|l|l}
0.0389 & 0.0379 & 0.0289 & 0.0269 & 0.0289 & 0.0339 & 0.024 & 0.0339 & 0.0379 & 0.0399 & 0.019 & 0.0329 & 0.024 & 0.0399 & 0.0459 & 0.0184 & 0.024 & 0.0319 & 0.024 & 0.0429 & 0 & 0.022 & 0.023 & 0.021 \\
\hline
\end{tabular} \begin{tabular}{|l|l|l|l|l|l|l|l|l|l|l|l|l|l|l|l|l|l|l|l|l|l|l|l|l|l|l}
0.0309 & 0.019 & 0.0339 & 0.0299 & 0.025 & 0.0279 & 0.019 & 0.023 & 0.0259 & 0.0279 & 0.0269 & 0.0329 & 0.024 & 0.024 & 0.0279 & 0.0349 & 0.0299 & 0.0329 & 0.021 & 0.0279 & 0.018 & 0 & 0.0379 & 0.0269
\end{tabular}

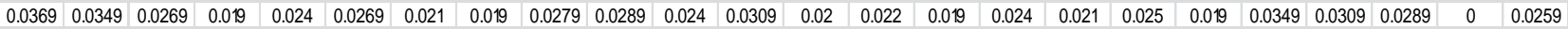

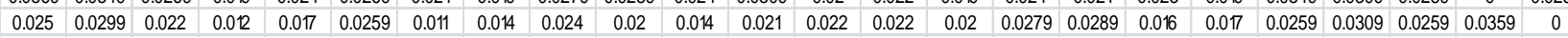

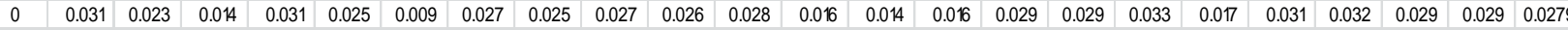

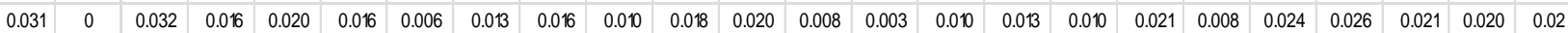

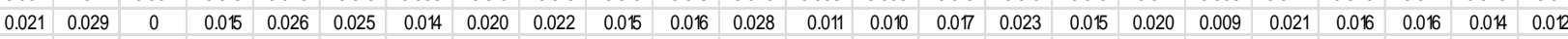

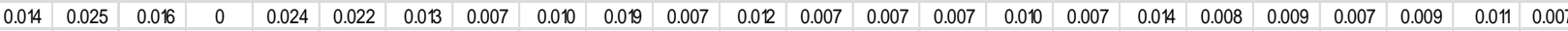

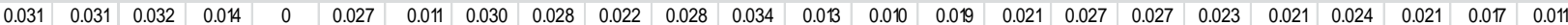

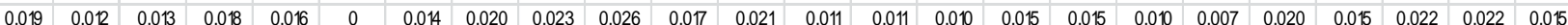
\begin{tabular}{|l|l|l|l|l|l|l|l|l|l|l|l|l|l|l|l|l|l|l|l|l|l|l|l|l|l|l|l|l|l|l|l|l|l|l|l|l|}
\hline 0.006 & 0.004 & 0.006 & 0.012 & 0.004 & 0.014 & 0 & 0.010 & 0.006 & 0.017 & 0.006 & 0.007 & 0.005 & 0.010 & 0.007 & 0.004 & 0.002 & 0.003 & 0.001 & 0.009 & 0.015 & 0.004 & 0.005 & 0.001 \\
\hline
\end{tabular}

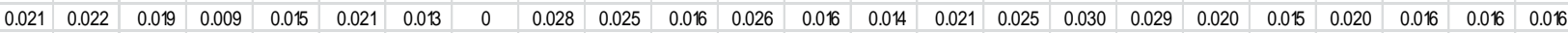

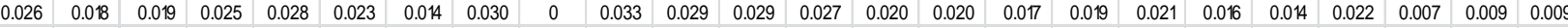

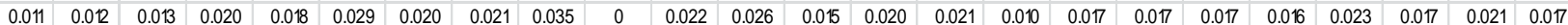

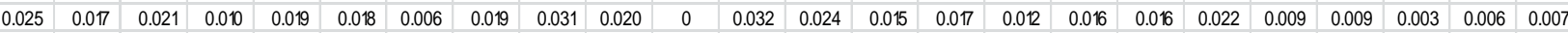

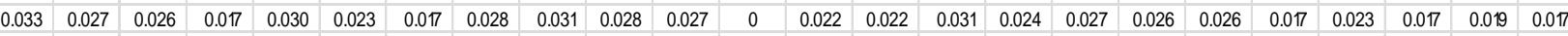

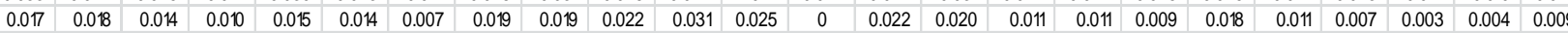

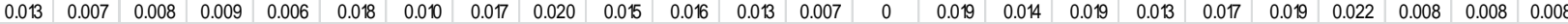

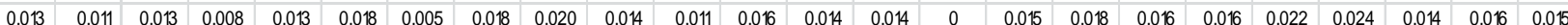

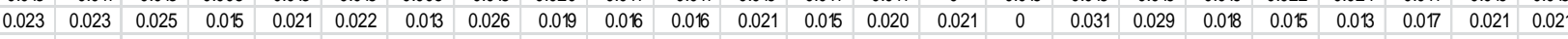

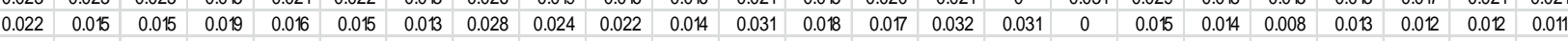

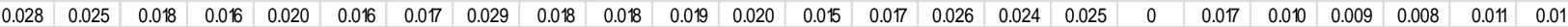

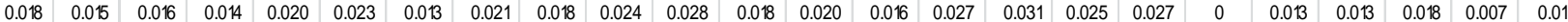
\begin{tabular}{|l|l|l|l|l|l|l|l|l|l|l|l|l|l|l|l|l|l|l|l|l|l|l|l|}
0.023 & 0.023 & 0.017 & 0.006 & 0.017 & 0.019 & 0.013 & 0.014 & 0.013 & 0.013 & 0.014 & 0.013 & 0.006 & 0.012 & 0.017 & 0.018 & 0.008 & 0.010 & 0.012 & 0 & 0.021 & 0.020 & 0.016 & 0.01 \\
\hline
\end{tabular}

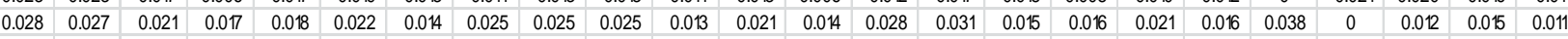

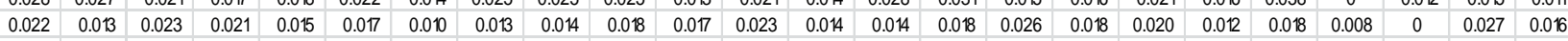

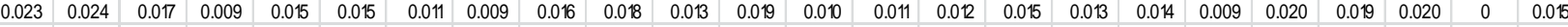
\begin{tabular}{|l|l|l|l|l|l|l|l|l|l|l|l|l|l|l|l|l|l|l|l|l|l|l|l|}
\hline 0.014 & 0.019 & 0.013 & 0.009 & 0.009 & 0.015 & 0.005 & 0.008 & 0.012 & 0.010 & 0.007 & 0.013 & 0.012 & 0.012 & 0.011 & 0.016 & 0.018 & 0.008 & 0.007 & 0.014 & 0.020 & 0.014 & 0.024 & 0 \\
\hline
\end{tabular}

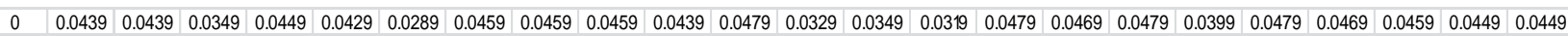

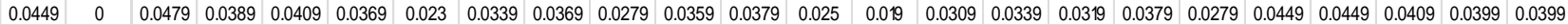
\begin{tabular}{l|l|l|l|l|l|l|l|l|l|l|l|l|l|l|l|l|l|l|l|l|l|l|l|l|l}
0.0429 & 0.0479 & 0 & 0.0349 & 0.0479 & 0.0459 & 0.0349 & 0.0429 & 0.0419 & 0.0339 & 0.0349 & 0.0469 & 0.0299 & 0.0319 & 0.0389 & 0.0459 & 0.0339 & 0.0419 & 0.0299 & 0.0409 & 0.0369 & 0.0349 & 0.0319 & 0.0299 \\
\hline
\end{tabular}

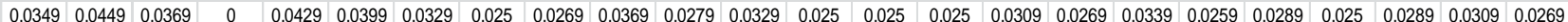

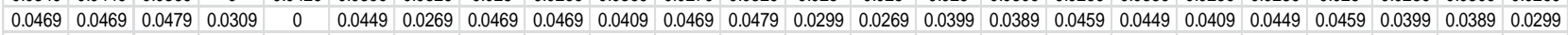

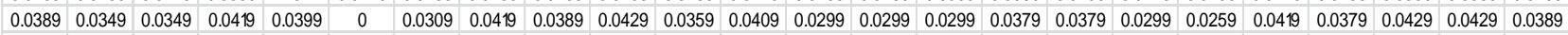

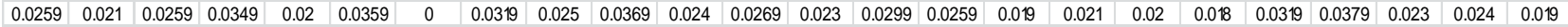
\begin{tabular}{l|l|l|l|l|l|l|l|l|l|l|l|l|l|l|l|l|l|l|l|l|l|l|l|l}
0.0419 & 0.0429 & 0.0409 & 0.0279 & 0.0369 & 0.0429 & 0.0349 & 0 & 0.0479 & 0.0409 & 0.0399 & 0.0469 & 0.0369 & 0.0349 & 0.0449 & 0.0459 & 0.0479 & 0.0479 & 0.0429 & 0.0339 & 0.0369 & 0.0329 & 0.0329 & 0.0339
\end{tabular}

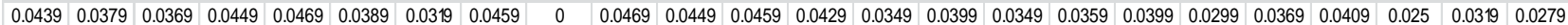

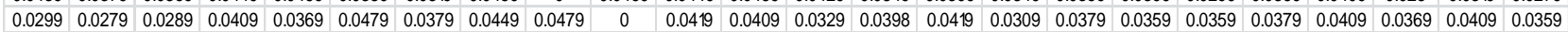

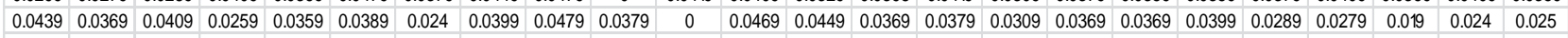

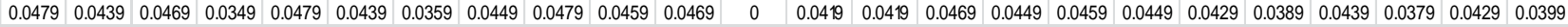

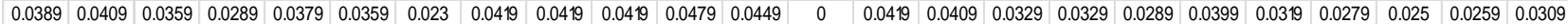

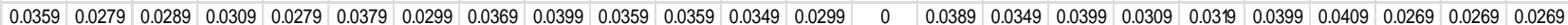

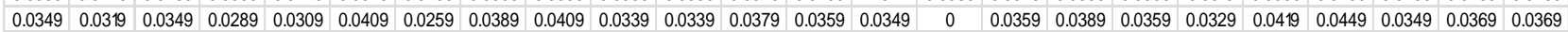

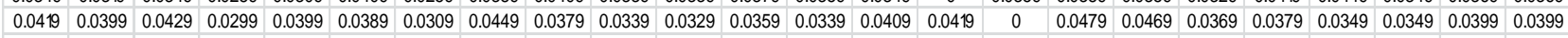
\begin{tabular}{|l|l|l|l|l|l|l|l|l|l|l|l|l|l|l|l|l|l|l|l|l|l|l|l|l|l|l|l|l|l}
0.0409 & 0.0349 & 0.0289 & 0.0369 & 0.0349 & 0.0289 & 0.0279 & 0.0449 & 0.0429 & 0.0399 & 0.0329 & 0.0459 & 0.0369 & 0.0349 & 0.0469 & 0.0469 & 0 & 0.0349 & 0.0299 & 0.0269 & 0.0289 & 0.0279 & 0.0279 & 0.0299 \\
\hline
\end{tabular} \begin{tabular}{l|l|l|l|l|l|l|l|l|l|l|l|l|l|l|l|l|l|l|l|l|l|l|l|l|l|l}
0.0429 & 0.0409 & 0.0379 & 0.0339 & 0.0359 & 0.0349 & 0.0349 & 0.0449 & 0.0379 & 0.0369 & 0.0389 & 0.0359 & 0.0309 & 0.0369 & 0.0459 & 0.0419 & 0.0439 & 0 & 0.0379 & 0.0309 & 0.0299 & 0.0309 & 0.0299 & 0.0289
\end{tabular}

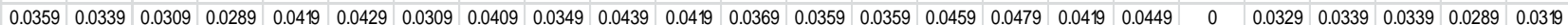

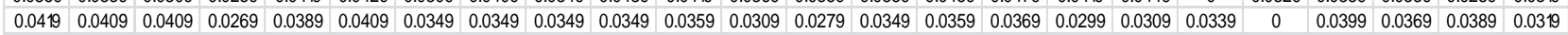

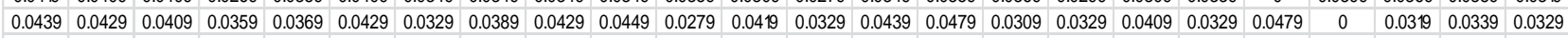
\begin{tabular}{l|l|l|l|l|l|l|l|l|l|l|l|l|l|l|l|l|l|l|l|l|l|l|l|l|l|l}
0.0429 & 0.0289 & 0.0439 & 0.0389 & 0.0349 & 0.0369 & 0.0299 & 0.0329 & 0.0349 & 0.0379 & 0.0389 & 0.0419 & 0.0359 & 0.0359 & 0.0399 & 0.0459 & 0.0389 & 0.0399 & 0.0329 & 0.0379 & 0.0279 & 0 & 0.0429 & 0.0359
\end{tabular} \begin{tabular}{l|l|l|l|l|l|l|l|l|l|l|l|l|l|l|l|l|l|l|l|l|l|l|l|l|l|l|l|l|l|l}
0.0429 & 0.0409 & 0.0379 & 0.0299 & 0.0339 & 0.0359 & 0.0309 & 0.0289 & 0.0379 & 0.0369 & 0.0359 & 0.0399 & 0.0309 & 0.0339 & 0.0289 & 0.0329 & 0.0309 & 0.0349 & 0.0309 & 0.0389 & 0.0389 & 0.0359 & 0 & 0.0359
\end{tabular}

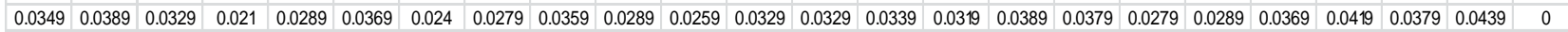


Appendix A5. The values of matrices $\left[l_{i j}^{\prime \prime}\right],\left[m_{i j}^{\prime \prime}\right]$ and $\left[r_{i j}^{\prime \prime}\right]$.

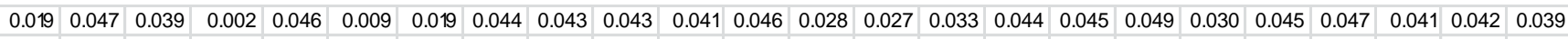
\begin{tabular}{ll|l|l|l|l|l|l|l|l|l|l|l|l|l|l|l|l|l|l|l|l|l|l}
0.043 & 0.012 & 0.043 & 0.009 & 0.031 & 0.006 & 0.013 & 0.025 & 0.028 & 0.021 & 0.028 & 0.033 & 0.016 & 0.012 & 0.021 & 0.024 & 0.021 & 0.032 & 0.017 & 0.034 & 0.036 & 0.029 & 0.029 & 0.028
\end{tabular}

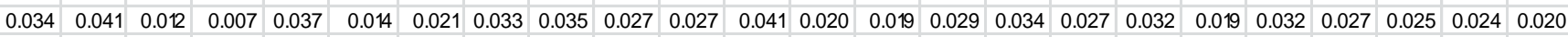

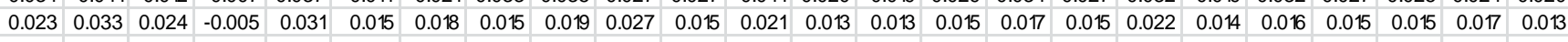
\begin{tabular}{l|l|l|l|l|l|l|l|l|l|l|l|l|l|l|l|l|l|l|l|l|l|l|l}
0.048 & 0.046 & 0.046 & 0.004 & 0.015 & 0.013 & 0.020 & 0.046 & 0.044 & 0.038 & 0.042 & 0.051 & 0.025 & 0.022 & 0.034 & 0.036 & 0.042 & 0.042 & 0.035 & 0.035 & 0.038 & 0.032 & 0.029 & 0.022 \\
\hline
\end{tabular}

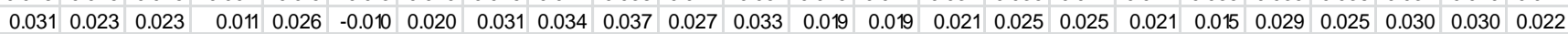

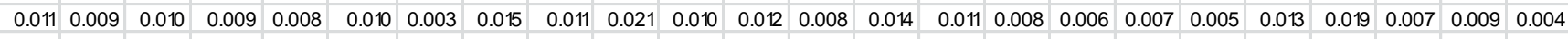

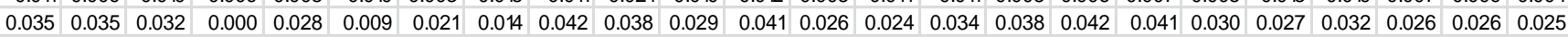
\begin{tabular}{l|l|l|l|l|l|l|l|l|l|l|l|l|l|l|l|l|l|l|l|l|l|l|l|l|l|l}
0.041 & 0.032 & 0.032 & 0.016 & 0.041 & 0.010 & 0.022 & 0.044 & 0.016 & 0.047 & 0.042 & 0.044 & 0.037 & 0.031 & 0.034 & 0.030 & 0.032 & 0.034 & 0.027 & 0.026 & 0.035 & 0.017 & 0.020 & 0.018 \\
\hline
\end{tabular}

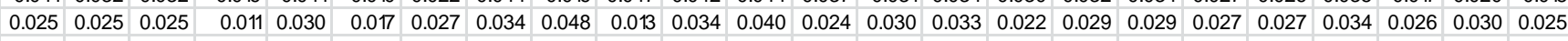

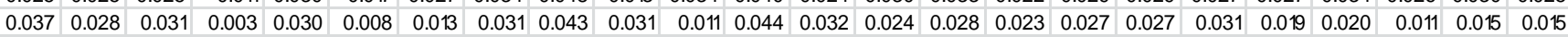

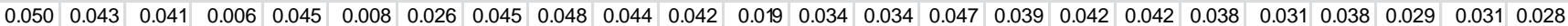

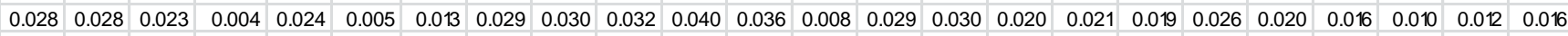

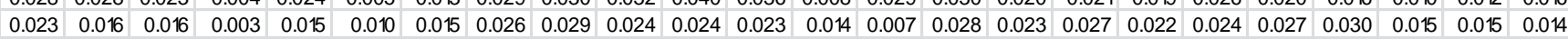
\begin{tabular}{l|l|l|l|l|l|l|l|l|l|l|l|l|l|l|l|l|l|l|l|l|l|l|l|l|l|l|l|l}
0.024 & 0.021 & 0.023 & 0.001 & 0.022 & 0.009 & 0.011 & 0.028 & 0.031 & 0.024 & 0.021 & 0.027 & 0.021 & 0.022 & 0.010 & 0.025 & 0.028 & 0.026 & 0.024 & 0.031 & 0.033 & 0.021 & 0.024 & 0.022 \\
\hline
\end{tabular}

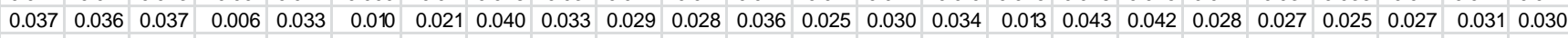

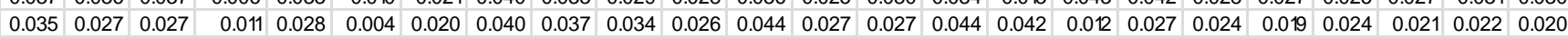

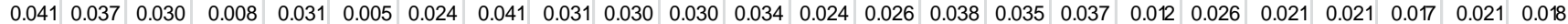

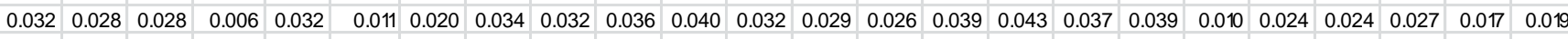

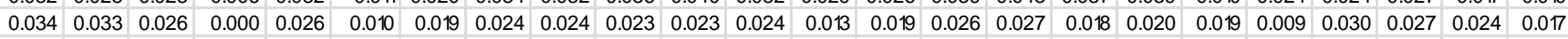

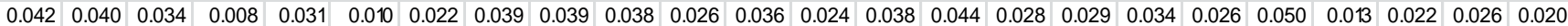

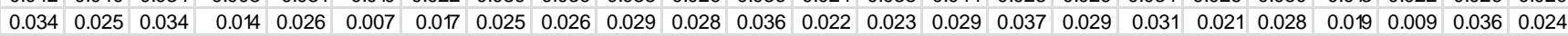

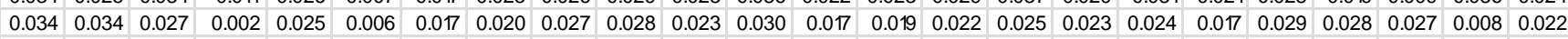

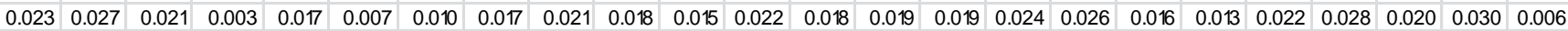

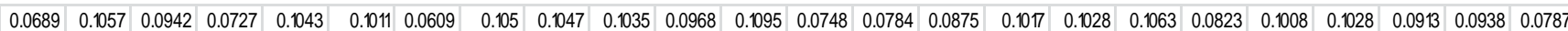

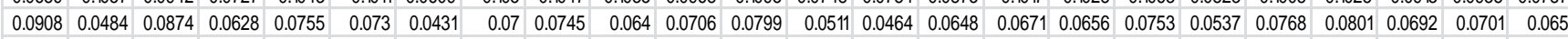
$\begin{array}{llllllllllllllllllllllllll}0.0849 & 0.0906 & 0.0483 & 0.0619 & 0.0861 & 0.0877 & 0.0569 & 0.083 & 0.0866 & 0.0724 & 0.0713 & 0.0939 & 0.0576 & 0.0615 & 0.0758 & 0.0824 & 0.072 & 0.0795 & 0.0556 & 0.0777 & 0.0721 & 0.0651 & 0.0642 & 0.057\end{array}$ $\begin{array}{lllllllllllllllllllllllllll}0.0598 & 0.0684 & 0.0588 & 0.0284 & 0.0692 & 0.0674 & 0.0462 & 0.052 & 0.0552 & 0.066 & 0.0501 & 0.0603 & 0.0421 & 0.0426 & 0.0461 & 0.0531 & 0.05 & 0.0576 & 0.0427 & 0.0492 & 0.047 & 0.046 & 0.0502 & 0.0405 \\ 0\end{array}$ \begin{tabular}{lllllllllllllllllllllllllll}
0.1062 & 0.1029 & 0.1 & 0.069 & 0.06 & 0.1002 & 0.0593 & 0.104 & 0.1037 & 0.0945 & 0.0965 & 0.1113 & 0.0701 & 0.0686 & 0.0895 & 0.0881 & 0.0974 & 0.1002 & 0.0822 & 0.0889 & 0.0901 & 0.0761 & 0.0772 & 0.0627 \\
\hline
\end{tabular} $\begin{array}{lllllllllllllllllllllllllllll}0.0791 & 0.0701 & 0.0671 & 0.066 & 0.0733 & 0.0489 & 0.0529 & 0.079 & 0.0795 & 0.0834 & 0.0699 & 0.0829 & 0.0552 & 0.0572 & 0.0641 & 0.0707 & 0.0714 & 0.0637 & 0.052 & 0.0736 & 0.0698 & 0.0709 & 0.0729 & 0.0612\end{array}$ $\begin{array}{lllllllllllllllllllllllllll}0.047 & 0.0376 & 0.0387 & 0.0434 & 0.0368 & 0.0521 & 0.0185 & 0.048 & 0.0414 & 0.0548 & 0.0369 & 0.0439 & 0.0318 & 0.041 & 0.04 & 0.0351 & 0.0347 & 0.0337 & 0.0265 & 0.0443 & 0.0505 & 0.0323 & 0.0343 & 0.0266\end{array}$ $\begin{array}{lllllllllllllllllllllllllll}0.0852 & 0.0858 & 0.076 & 0.0579 & 0.0767 & 0.0862 & 0.0593 & 0.057 & 0.0933 & 0.0891 & 0.0784 & 0.0958 & 0.0665 & 0.0687 & 0.0856 & 0.0877 & 0.0934 & 0.092 & 0.0736 & 0.0736 & 0.0796 & 0.0673 & 0.0693 & 0.0612\end{array}$

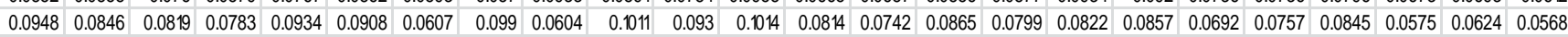
$\begin{array}{lllllllllllllllllllllllllll}0.0708 & 0.0692 & 0.067 & 0.0705 & 0.0765 & 0.0927 & 0.0636 & 0.088 & 0.1002 & 0.0537 & 0.0809 & 0.0924 & 0.0658 & 0.0737 & 0.0821 & 0.07 & 0.0775 & 0.0753 & 0.0679 & 0.0698 & 0.0812 & 0.0679 & 0.0719 & 0.0605\end{array}$

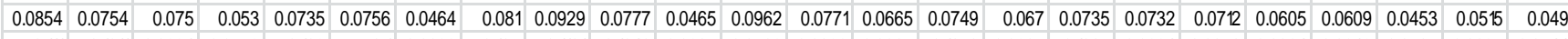
$\begin{array}{llllllllllllllllllllllllllll}0.111 & 0.1011 & 0.0934 & 0.0753 & 0.103 & 0.1 & 0.0699 & 0.105 & 0.1101 & 0.1046 & 0.0987 & 0.0709 & 0.0832 & 0.0867 & 0.1058 & 0.0967 & 0.1007 & 0.0994 & 0.0878 & 0.0864 & 0.0943 & 0.0785 & 0.0837 & 0.0744\end{array}$ $\begin{array}{llllllllllllllllllllllllll}0.075 & 0.0742 & 0.0668 & 0.0524 & 0.0704 & 0.072 & 0.0422 & 0.078 & 0.0798 & 0.0803 & 0.0857 & 0.0867 & 0.0372 & 0.0706 & 0.0768 & 0.0669 & 0.0679 & 0.0638 & 0.0669 & 0.0618 & 0.0602 & 0.0495 & 0.0521 & 0.0534\end{array}$ \begin{tabular}{lllllllllllllllllllllllll}
0.0679 & 0.058 & 0.0563 & 0.0525 & 0.057 & 0.0718 & 0.0476 & 0.072 & 0.0762 & 0.07 & 0.0663 & 0.0708 & 0.0516 & 0.0357 & 0.0721 & 0.065 & 0.0705 & 0.0616 & 0.0586 & 0.0678 & 0.0679 & 0.0484 & 0.05 & 0.0458 \\
\hline
\end{tabular} $\begin{array}{lllllllllllllllllllllllllll}0.0713 & 0.066 & 0.0657 & 0.0535 & 0.0664 & 0.0765 & 0.0453 & 0.076 & 0.0801 & 0.0709 & 0.0651 & 0.0777 & 0.0611 & 0.0632 & 0.0458 & 0.0697 & 0.0742 & 0.07 & 0.0606 & 0.0734 & 0.078 & 0.0592 & 0.0629 & 0.0582 \\ \end{array}$ $\begin{array}{llllllllllllllllllllllllll}0.0923 & 0.0895 & 0.0878 & 0.0635 & 0.0857 & 0.0877 & 0.0591 & 0.094 & 0.089 & 0.081 & 0.0758 & 0.0908 & 0.0682 & 0.077 & 0.0856 & 0.0537 & 0.0966 & 0.0905 & 0.0717 & 0.0762 & 0.0745 & 0.0697 & 0.0783 & 0.0727\end{array}$ $\begin{array}{llllllllllllllllllllllllll}0.0835 & 0.0753 & 0.0666 & 0.066 & 0.0741 & 0.073 & 0.0526 & 0.09 & 0.0883 & 0.0833 & 0.071 & 0.0962 & 0.0672 & 0.0692 & 0.0918 & 0.0894 & 0.0494 & 0.0733 & 0.0613 & 0.0606 & 0.0666 & 0.0584 & 0.0604 & 0.0546\end{array}$

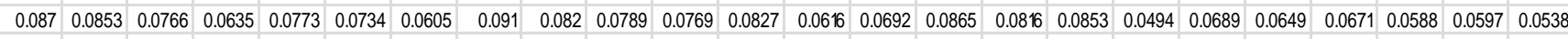
$\begin{array}{lllllllllllllllllllllllllll}0.0824 & 0.0776 & 0.0717 & 0.0609 & 0.0874 & 0.0875 & 0.0577 & 0.087 & 0.0824 & 0.0884 & 0.0882 & 0.0868 & 0.0717 & 0.071 & 0.0905 & 0.0927 & 0.0883 & 0.0905 & 0.0434 & 0.071 & 0.073 & 0.0685 & 0.0611 & 0.0587\end{array}$

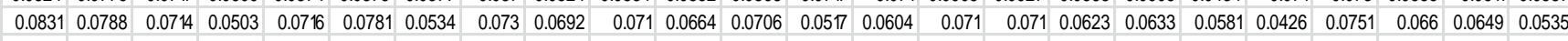
$\begin{array}{llllllllllllllllllllllllllll}0.0954 & 0.0916 & 0.0798 & 0.0691 & 0.0817 & 0.0897 & 0.0609 & 0.09 & 0.0952 & 0.0949 & 0.0709 & 0.0919 & 0.0668 & 0.0843 & 0.0983 & 0.0709 & 0.077 & 0.0843 & 0.0677 & 0.0923 & 0.0523 & 0.0657 & 0.0686 & 0.061\end{array}$ $\begin{array}{lllllllllllllllllllllllll}0.0823 & 0.0686 & 0.0794 & 0.0674 & 0.0726 & 0.0781 & 0.0524 & 0.074 & 0.0779 & 0.0778 & 0.0729 & 0.0861 & 0.0626 & 0.0647 & 0.0758 & 0.0815 & 0.0775 & 0.0799 & 0.0604 & 0.0726 & 0.064 & 0.0396 & 0.0782 & 0.0627\end{array}$

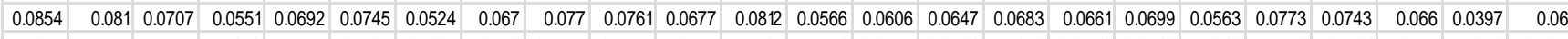

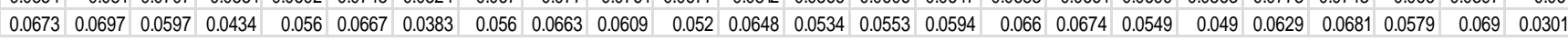

\begin{tabular}{|c|c|c|c|c|c|c|c|c|c|c|c|c|c|c|c|c|c|c|c|c|c|c|c|}
\hline 47 & 77 & 76 & 39 & 76 & 886 & 217 & 286 & 91 & 0.282 & 71 & 90 & $3 r$ & 47 & r & 79 & 0278 & 0278 & 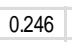 & 77 & 75 & 551 & 60 & 24 \\
\hline & & & & & & & & & & & & & & & & & & & & & & & 21 \\
\hline 64 & & & & 56 & & & & & & & & & & & & & & & & 244 & & 227 & 213 \\
\hline & 15 & & & 21 & & & & & & & & & & & & & & & & & & 189 & .176 \\
\hline & & & & & & & & & & & & & & & & & & & & & & & \\
\hline 251 & 37 & 36 & 218 & 40 & & & & & & & & & 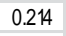 & & & & & & & & 220 & 229 & \\
\hline 177 & & & & & & & & & & & & & .101 & .17 & 10 & 104 & & & & & 50 & $5 /$ & 140 \\
\hline & & & & & & & & & & & & & & & & & & & & & & & \\
\hline & 4 & 47 & & & & 0.4 & & & & & & & & & & & & & & & & 227 & 212 \\
\hline & & & & & & & & & & & & & & & & & & & & & & & 216 \\
\hline & & & & & & & & & & & & & & & & & & & & & & & \\
\hline 96 & 80 & 82 & 45 & & & 0.226 & & & & & & & 25 & & & & & & & & 247 & 261 & 246 \\
\hline & & & & & & & & & & & & & & & & & & & & & & & 202 \\
\hline & & & & & & & & & & & & & & & & & & & & & & & .187 \\
\hline 42 & & & 0 & & & & & & & & & & & & 0.2 & 2 & & & & & & 218 & 208 \\
\hline & & & & & & & & & & & & & & & & & & & & & 21 & & 223 \\
\hline & & & & & & & & & & & & & & & & & & & & & & & \\
\hline 254 & & & & & & & & & & & & & & & & & & & & & & 215 & 204 \\
\hline & & & & & & & & & & & & & & & & & & & & & & & \\
\hline & & & & & & & & & & & & & & & & & & & & & & & \\
\hline & & & & & 0.2 & 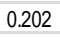 & & & & & & & & & & 0.2 & & & & & 217 & 228 & 216 \\
\hline & & & & & & & & & & & & & & & & & & & & & & & 113 \\
\hline & & & & & & & & & & & & & & & & & & & & & & & \\
\hline & & & IVL & 0.210 & $-t$ & 0.117 & $0.2 \pi$ &.$\angle C T$ & $0.2 \pi$ & $\angle 0 J$ & 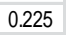 & & 200 & & 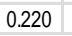 & ch & 20 & & 2 & $2<0$ & 198 & .212 & 100 \\
\hline
\end{tabular}


Appendix A6. The total-relation fuzzy matrix $\tilde{T}$.

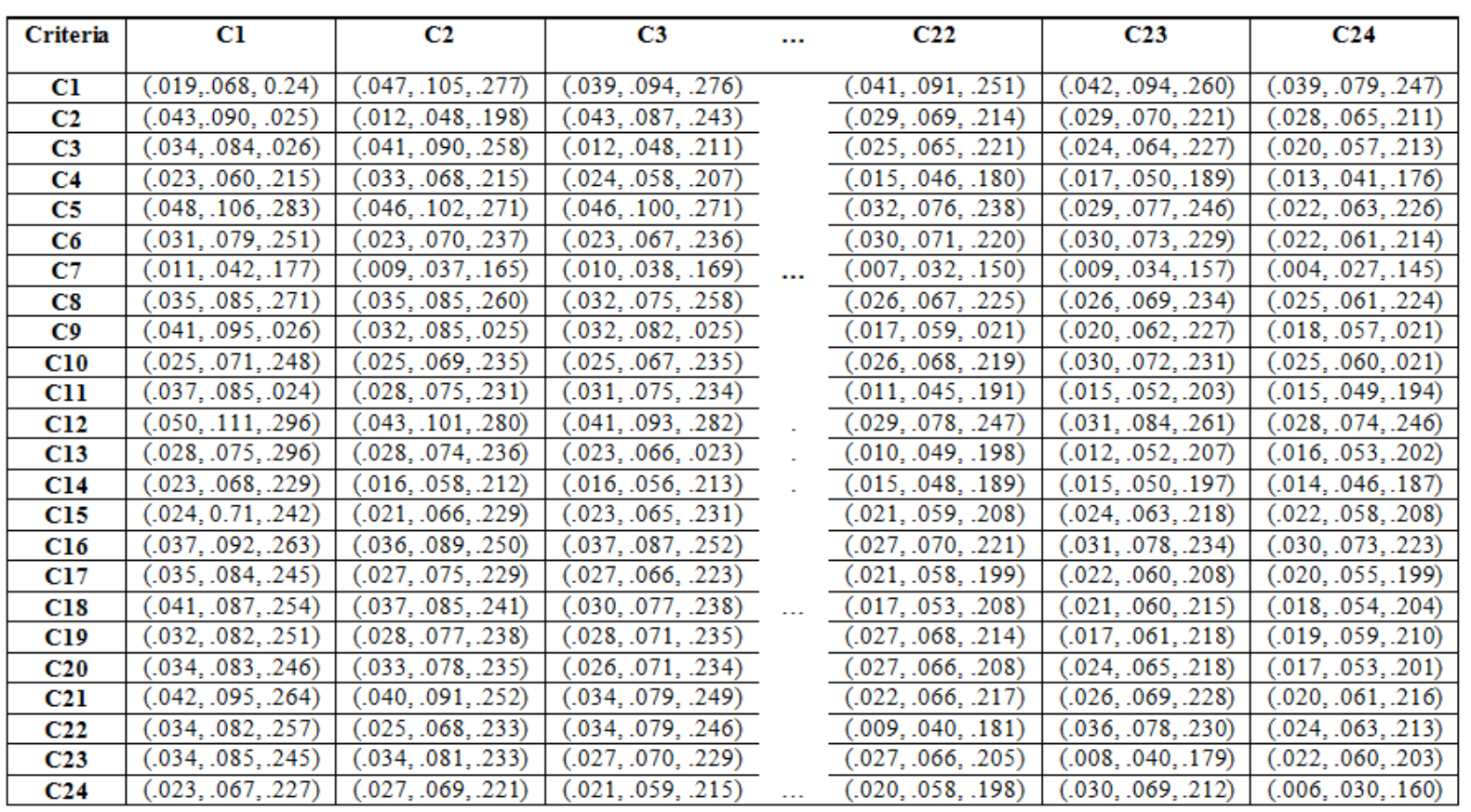

